\title{
NOUVELLES PROPRIÉTÉS DES COURBES ET RELATION DE DISPERSION EN ÉLASTICITÉ LINÉAIRE
}

\author{
TARK Bouhennache ${ }^{1}$ Et Yves DeRmenjian ${ }^{1}$
}

\begin{abstract}
In the case of an elastic strip we exhibit two properties of dispersion curves $\lambda_{n}, n \geq 1$, that were not pointed out previously. We show cases where $\lambda_{n}^{\prime}(0)=\lambda_{n}^{\prime \prime}(0)=\lambda_{n}^{\prime \prime \prime}(0)=0$ and we point out that these curves are not automatically monotoneous on $\mathbb{R}_{+}$. The non monotonicity was an open question (see [2], for example) and, for the first time, we give a rigourous answer. Recall the characteristic property of the dispersion curves: $\left\{\lambda_{n}(p) ; n \geq 1\right\}$ is the set of eigenvalues of $A_{p}$, counted with their multiplicity. The operators $A_{p}, p \in \mathbb{R}$, are the reduced operators deduced from the elastic operator $A$ using a partial Fourier transform. The second goal of this article is the introduction of a dispersion relation $D(p, \lambda)=0$ in a general framework, and not only for a homogeneous situation (in this last case the relation is explicit). Recall that a dispersion relation is an implicit equation the solutions of which are eigenvalues of $A_{p}$. The main property of the function $D$ that we build is the following one: the multiplicity of an eigenvalue $\lambda$ of $A_{p}$ is equal to the multiplicity it has as a root of $D(p, \lambda)=0$. We give also some applications.
\end{abstract}

Résumé. Dans cet article nous exhibons deux propriétés des courbes de dispersion $\lambda_{n}, n \geq 1$, associées à une bande élastique, qui n'ont pas été mises en évidence jusqu'à maintenant : nous montrons des situations où $\lambda_{n}^{\prime}(0)=\lambda_{n}^{\prime \prime}(0)=\lambda_{n}^{\prime \prime \prime}(0)=0$ et, de plus, nous montrons que ces courbes ne sont pas systématiquement monotones sur $\mathbb{R}_{+}$. La non monotonie était un problème ouvert (il a été posé dans un contexte différent dans [2]) auquel nous répondons pour la première fois. Rappelons que les courbes de dispersion sont telles que, pour tout $p \in \mathbb{R},\left\{\lambda_{n}(p) ; n \geq 1\right\}$ est l'ensemble des valeurs propres de $A_{p}$, comptées avec leur ordre de multiplicité. Les opérateurs $A_{p}, p \in \mathbb{R}$, sont les opérateurs réduits obtenus en utilisant la transformation de Fourier partielle. Un deuxième objectif pour cet article est d'introduire une relation de dispersion $D(p, \lambda)=0$ dans un cadre général, et pas seulement dans le cas homogène où elle est calculée explicitement. Rappelons qu'une relation de dispersion est une équation implicite donnant la condition pour que $\lambda$ soit une valeur propre de $A_{p}$. Nous montrons la principale propriété de la fonction $D$ que nous construisons : la multiplicité d'une valeur propre $\lambda$ de $A_{p}$ est égale à sa multiplicité comme racine de $D(p, \lambda)=0$ et nous présentons quelques applications.

AMS Subject Classification. 73D25, 73D20, 35P.

Reçu : 10 août 1998. Révisé : 8 février 1999.

Keywords and phrases. Élasticité, relation de dispersion, valeur propre.

${ }^{1}$ CMI, 39 rue Joliot Curie, 13453 Marseille Cedex 13, France.

e-mail: dermenji@gyptis.univ-mrs.fr, bouhenn@gyptis.univ-mrs.fr 


\section{INTRODUCTION ET PRINCIPAUX RÉSULTATS}

La notion de relation de dispersion apparaît dans diverses disciplines (mécanique, géophysique, ... ), souvent avec des sens légèrement différents. Nous illustrerons celle-ci par deux exemples.

Considérons tout d'abord un opérateur aux dérivées partielles $P(D)$ d'ordre $m$, et à coefficients constants dans l'ouvert $\vartheta \subset \mathbb{R}^{n}$. Si $\alpha=\left(\alpha_{1}, \ldots, \alpha_{n}\right)$ est un multi-indice, i.e. $\alpha \in \mathbb{N}^{n}$, on pose $|\alpha|=\alpha_{1}+\ldots+\alpha_{n}$ et l'élément générique de $\vartheta$ est noté $x=\left(x_{1}, \ldots, x_{n}\right)$. On peut alors écrire

$$
P(D)=\sum_{|\alpha| \leq m} a_{\alpha} i^{-\alpha} \frac{\partial^{|\alpha|}}{\partial x^{\alpha}}
$$

et lui faire correspondre le polynôme à $n$ variables $P(\xi)=\sum_{|\alpha|<m} a_{\alpha} \xi^{\alpha}$, où $\xi \in \mathbb{R}^{n}$ et $\xi^{\alpha}:=\xi_{1}^{\alpha_{1}} \cdots \xi_{n}^{\alpha_{n}}$.

Lorsque l'ouvert $\vartheta$ est convexe, que le polynôme $P$ n'a pas de facteur multiple et que la distribution $f$ appartient à un espace de Besov local, la résolution de l'équation $P(D) u=f$ peut se faire à l'aide de ce que l'on appelle les ondes planes, i.e. les fonctions $x \longrightarrow \mathrm{e}^{i x . \xi}\left(x . \xi:=\sum_{j=1}^{n} x_{j} \xi_{j}\right)$. On n'a effectivement besoin que de celles vérifiant $P(D) \mathrm{e}^{i x . \xi}=0$ (cf. [8], Chap. X, et aussi [11]) ce qui signifie que le paramètre $\xi \in \mathbb{R}^{n}$ doit satisfaire

$$
P(\xi)=0 .
$$

Cette équation est appelée relation de dispersion et, dans le cas présent, ses solutions se confondent avec l'ensemble caractéristique de $P(D)$. Le théorème des fonctions implicites appliqué à $(1)$ permet de définir des fonctions $\lambda$ donnant une coordonnée en fonction des autres. On pourra ainsi avoir l'application $\xi^{\prime}=$ $\left(\xi_{1}, \ldots, \xi_{n-1}\right) \longrightarrow \lambda\left(\xi_{1}, \ldots, \xi_{n-1}\right)=\xi_{n}$ vérifiant $P\left(\xi_{1}, \ldots, \xi_{n-1}, \xi_{n}\right)=0$. Une telle application $\lambda$ est un exemple de courbe de dispersion. Dans le cas particulier $P(D)=\partial_{x_{n}}^{2}-\sum_{1 \leq j \leq n-1} \frac{\partial^{2}}{\partial x_{j}^{2}}$ on aura donc les deux courbes $\omega_{+}\left(\xi^{\prime}\right)=\left|\xi^{\prime}\right|$ et $\omega_{-}\left(\xi^{\prime}\right)=-\left|\xi^{\prime}\right|$ en posant $\lambda=\omega_{ \pm}^{2}$. Si $n=4$, le géophysicien préférera fixer la fréquence $\omega=\xi_{4}$ $\left(\omega^{2}=\lambda\right)$ et considérer la courbe de dispersion $\left(\xi_{1}, \xi_{2}\right) \longrightarrow \xi_{3}\left(\xi_{1}, \xi_{2}, \omega\right)$.

Nous allons retrouver ces deux notions pour des opérateurs à coefficients dans $L^{\infty}$ et elles nous permettront d'éviter les techniques microlocales usuelles (qui seraient d'ailleurs inapplicables telles quelles ici). Supposons que l'ouvert $\vartheta$ soit de la forme $\Omega \times \mathbb{R}$ et que l'opérateur $P(D)$ corresponde à une équation des ondes, $i . e$. $P(D)=\partial_{x_{n}}^{2}+P\left(x^{\prime}, D^{\prime}\right)$ où $P\left(x^{\prime}, D^{\prime}\right)$ représente un opérateur aux dérivées partielles à coefficients variables qui ne met en jeu que les premières coordonnées $x^{\prime}=\left(x_{1}, \ldots, x_{n-1}\right)$ (on peut penser $x_{n}$ comme le temps). Si $P\left(x^{\prime}, D^{\prime}\right)$ admet une extension autoadjointe dans $L^{2}\left(\mathbb{R}^{n-1}\right)$, une approche consiste à faire l'étude spectrale de l'opérateur "stationnaire" $P\left(x^{\prime}, D^{\prime}\right)$. Celle-ci est difficile à réaliser de façon explicite dans le cas général mais possible si le milieu est stratifié.

Pour fixer les idées considérons

$$
P\left(x^{\prime}, D^{\prime}\right)=-\nabla \cdot c^{2}\left(x_{n-1}\right) \nabla
$$

et $\Omega=\mathbb{R}^{n-2} \times(0, L)$ (avec des conditions aux bords pour $x_{n-1}=0$ et $\left.x_{n-1}=L\right)$. Une transformation de Fourier partielle par rapport à $x^{\prime \prime}=\left(x_{1}, \ldots, x_{n-2}\right), p$ étant la variable duale de $x^{\prime \prime}$, introduit une famille d'opérateurs différentiels réduits $P_{p}\left(x_{n-1}, \partial_{n-1}\right)$ à coefficients dans $L^{\infty}, P_{p}=-\frac{\mathrm{d}}{\mathrm{d} x_{n-1}}\left(c^{2}\left(x_{n-1}\right) \frac{\mathrm{d}}{\mathrm{d} x_{n-1}}\right)+c^{2}\left(x_{n-1}\right)|p|^{2}$. Le spectre de cet opérateur est souvent connu et fait intervenir des fonctions $p \longrightarrow \lambda(|p|)$ donnant les valeurs propres de l'opérateur différentiel $P_{p}$. Elles permettent de décrire cet opérateur, complètement ou non selon les cas, en utilisant le théorème spectral qui est en fait un théorème de représentation de l'espace d'Hilbert $L^{2}(\Omega)$ dans lequel on travaille. Ces courbes sont un autre exemple de courbes de dispersion et sont solutions d'une équation implicite qui est une relation de dispersion. C'est cette approche que nous illustrerons dans cet article.

Notons encore qu'il est facile de construire des opérateurs abstraits avec des courbes de dispersion prescrites mais ce n'est plus le cas si l'on exige que ces opérateurs soient des opérateurs aux dérivées partielles. En effet, 
les exemples étudiés de ce point de vue jusqu'à maintenant ont toujours des courbes monotones en $|p|$. Parmi d'autres choses nous exhiberons des contre-exemples.

Nous considérons à partir de maintenant une bande $\Omega=\left\{\mathbf{x}=\left(x_{1}, x_{2}\right) ; x_{2} \in(0, L)\right\} \subset \mathbb{R}^{2}$. Étant élastique, isotrope et stratifiée, elle est caractérisée par sa densité $\rho$ et ses coefficients de Lamé $\underline{\lambda}$ et $\mu$ qu'on suppose être des fonctions mesurables telles qu'il existe deux constantes $m, m^{\prime}>0$ avec $m \leq \underline{\rho}(\mathbf{x}), \underline{\lambda}(\mathbf{x}), \underline{\mu}(\mathbf{x}) \leq m^{\prime}$, p.p. $\mathbf{x} \in \Omega$ (i.e. pour presque tout $\mathbf{x} \in \Omega$ ). Le fait que la bande $\Omega$ soit stratifiée se traduit par la non dépendance de $\underline{\rho}, \underline{\lambda}$ et $\underline{\mu}$ en $x_{1}$. Le cas le plus simple est celui de la bande homogène dans laquelle $\underline{\rho}, \underline{\lambda}$ et $\underline{\mu}$ sont des constantes positives. L'opérateur de l'élasticité linéaire sur $\Omega$ est donné formellement par

$$
(\mathcal{A} \mathbf{u})_{i}=-\frac{1}{\rho} \sum_{j=1}^{2} \frac{\partial \sigma_{i j}(\mathbf{u})}{\partial x_{j}}, \quad \text { avec } \quad \sigma_{i j}(\mathbf{u})=\underline{\lambda}(\nabla \cdot \mathbf{u}) \delta_{i j}+\underline{\mu}\left(\frac{\partial u_{i}}{\partial x_{j}}+\frac{\partial u_{j}}{\partial x_{i}}\right), 1 \leq i, j \leq 2,
$$

où $\mathbf{u}=\left(u_{1}, u_{2}\right)$ est le champ de déplacement. Notons $\mathbf{V}=\left\{\mathbf{u} \in H^{1}\left(\Omega, \mathbb{C}^{2}\right) ; \mathbf{u}_{\mid x_{2}=L}=0\right\}$ et $H$ l'espace de Hilbert $L^{2}\left(\Omega, \mathbb{C}^{2}\right)$ muni du produit scalaire $\langle\mathbf{u}, \mathbf{v}\rangle_{H}=\int_{\Omega} \sum_{1}^{2} u_{i} \overline{v_{i}} \rho \mathrm{d} \mathbf{x}$. L'opérateur auto-adjoint $A$ dans $H$ et associé à la bande élastique avec la condition de surface libre en $x_{2}=0$ et celle de Dirichlet en $x_{2}=L$, est défini par

$$
\left\{\begin{array}{l}
\mathcal{D}(A)=\left\{\mathbf{u} \in \mathbf{V} ; \mathcal{A} \mathbf{u} \in H, \sigma_{12}(\mathbf{u})_{\mid x_{2}=0}=\sigma_{22}(\mathbf{u})_{\mid x_{2}=0}=0\right\} \\
A \mathbf{u}=\mathcal{A} \mathbf{u}, \quad \forall \mathbf{u} \in \mathcal{D}(A) .
\end{array}\right.
$$

Ce dernier a été étudié dans [4] (voir aussi [3]), où on voit que ses propriétés spectrales dépendent d'une famille de fonctions $\lambda_{n}, n \geq 1$, représentant les courbes de dispersion. Nous allons ci-dessous suivre la même démarche pour introduire ces fonctions.

La transformation de Fourier partielle $\mathcal{F}$ par rapport à $x_{1}$ est définie, pour tout $\mathbf{u} \in H$, par

$$
\mathcal{F} \mathbf{u}\left(p, x_{2}\right)=\text { l.i.m. }(2 \pi)^{-1 / 2} \int_{\mathbb{R}} \mathbf{u}\left(x_{1}, x_{2}\right) \mathrm{e}^{-i p x_{1}} \mathrm{~d} x_{1}, \quad \text { p.p. }\left(p, x_{2}\right) \in \Omega,
$$

et elle est unitaire dans $H$. Comme $\underline{\rho}, \underline{\lambda}$ et $\underline{\mu}$ ne dépendent pas de $x_{1}$, on vérifie que l'opérateur $\widehat{A}$, unitairement équivalent à $A$ et défini par $\mathcal{F} A \mathcal{F}^{-1}$ avec $\mathcal{D}(\widehat{A})=\mathcal{F} \mathcal{D}(A)$, est l'intégrale directe du champs d'opérateurs réduits $A_{p}, p \in \mathbb{R}$, définis ci-dessous, i.e. $\widehat{A}=\int_{\mathbb{R}}^{\oplus} A_{p} \mathrm{~d} p$.

Dorénavant, pour simplifier, nous noterons par $y$ la variable $x_{2} \in(0, L)$. Pour tout $p \in \mathbb{R}$ fixé, considérons l'opérateur différentiel

$$
\mathcal{A}_{p} \mathbf{u}=-\frac{1}{\underline{\rho}}\left(\begin{array}{c}
-p^{2}(\underline{\lambda}+2 \underline{\mu}) u_{1}+i p \underline{\lambda} \frac{\mathrm{d} u_{2}}{\mathrm{~d} y}+\frac{\mathrm{d}}{\mathrm{d} y}\left(\underline{\mu}\left(i p u_{2}+\frac{\mathrm{d}}{\mathrm{d} y} u_{1}\right)\right) \\
-p^{2} \underline{\mu} u_{2}+i p \underline{\mu} \frac{\mathrm{d} u_{1}}{\mathrm{~d} y}+\frac{\mathrm{d}}{\mathrm{d} y}\left((\underline{\lambda}+2 \underline{\mu}) \frac{\mathrm{d} u_{2}}{\mathrm{~d} y}+i p \underline{\lambda} u_{1}\right)
\end{array}\right) .
$$

Notons $\overline{\mathbf{V}}=\left\{\mathbf{u} \in H^{1}\left((0, L), \mathbb{C}^{2}\right) ; \mathbf{u}(L)=0\right\}$ et $\bar{H}$ l'espace de Hilbert $L^{2}\left((0, L), \mathbb{C}^{2}\right)$ muni du produit scalaire $\langle\mathbf{u}, \mathbf{v}\rangle_{\bar{H}}=\int_{0}^{L} \sum_{1}^{2} u_{i} \overline{v_{i}} \underline{\rho} \mathrm{d} y$. L'opérateur $A_{p}$ est défini par

$$
\left\{\begin{array}{l}
\mathcal{D}\left(A_{p}\right)=\left\{\mathbf{u} \in \overline{\mathbf{V}} ; \mathcal{A}_{p} \mathbf{u} \in \bar{H}, \underline{\mu}\left(\frac{\mathrm{d} u_{1}}{\mathrm{~d} y}+i p u_{2}\right)_{\mid y=0}=0,\left(i \underline{\lambda} p u_{1}+(\underline{\lambda}+2 \underline{\mu}) \frac{\mathrm{d} u_{2}}{\mathrm{~d} y}\right)_{\mid y=0}=0\right\} \\
A_{p} \mathbf{u}=\mathcal{A}_{p} \mathbf{u}, \quad \forall \mathbf{u} \in \mathcal{D}\left(A_{p}\right) .
\end{array}\right.
$$


C'est un opérateur auto-adjoint associé à la forme hermitienne, bornée et $\overline{\mathbf{V}}$-coercive par rapport à $\bar{H}$, définie par

$$
\begin{aligned}
a_{p}(\mathbf{u}, \mathbf{v}):= & \int_{0}^{L}\left[\underline{\lambda}\left(i p u_{1}+\frac{\mathrm{d} u_{2}}{\mathrm{~d} y}\right) \overline{\left(i \bar{p} v_{1}+\frac{\mathrm{d} v_{2}}{\mathrm{~d} y}\right)}+2 \underline{\mu}\left(i p u_{1}\right) \overline{\left(i \bar{p} v_{1}\right)}\right. \\
& \left.+2 \underline{\mu} \frac{\mathrm{d} u_{2}}{\mathrm{~d} y} \frac{\overline{\mathrm{d} v_{2}}}{\mathrm{~d} y}+\underline{\mu}\left(i p u_{2}+\frac{\mathrm{d} u_{1}}{\mathrm{~d} y}\right) \overline{\left(i \bar{p} v_{2}+\frac{\mathrm{d} v_{1}}{\mathrm{~d} y}\right)}\right] \mathrm{d} y, \quad \forall \mathbf{u}, \mathbf{v} \in \overline{\mathbf{V}} .
\end{aligned}
$$

L'écriture ci-dessus montre que la définition de $a_{p}(\cdot, \cdot)$ s'étend à tout $p \in \mathbb{C}$. On obtient ainsi une famille holomorphe auto-adjointe de type $(a)$ ( $c f$. [9], Chap. VII). Comme pour $p$ réel, on vérifie que $a_{p}(\cdot, \cdot)$ est $\overline{\mathbf{V}}$-coercive par rapport à $\bar{H}$ pour tout $p \in \mathbb{C}$. L'injection $\overline{\mathbf{V}} \hookrightarrow \bar{H}$ étant compacte on en déduit que l'opérateur $A_{p}$, associé à $a_{p}(\cdot, \cdot)$, a une résolvante compacte.

Donc $A_{p}, p \in \mathbb{C}$, est une famille holomorphe auto-adjointe de type $(B)$ et on peut appliquer la remarque 4.22 du chapitre VII de [9] donnant l'existence de deux familles de fonctions analytiques sur $\mathbb{R}$, à savoir $p \longrightarrow$ $\lambda_{n}(p) \in \mathbb{R}$ et $p \longrightarrow W_{n}(p) \in H, n \geq 1$. Pour tout $p \in \mathbb{R},\left\{\lambda_{n}(p) ; n \geq 1\right\}$ est l'ensemble des valeurs propres de $A_{p}$ comptées avec leur ordres de multiplicité, et $\left\{W_{n}(p) ; n \geq 1\right\}$ est une base orthonormée de vecteurs propres associés.

L'opérateur $A$ est unitairement équivalent à l'opérateur de multiplication $M$ dans $\oplus_{1}^{\infty} L^{2}(\mathbb{R}):=$ $\left\{\left(f^{n}\right)_{n \geq 1} ; \sum_{1}^{\infty}\left\|f^{n}\right\|_{L^{2}(\mathbb{R})}^{2}<\infty\right\}$ par les fonctions $\lambda_{n}, n \geq 1$. Ce dernier est défini par

$$
\left\{\begin{array}{l}
\mathcal{D}(M)=\left\{\left(f^{n}\right)_{n \geq 1} \in \oplus_{1}^{\infty} L^{2}(\mathbb{R}) ;\left(\lambda_{n} f^{n}\right)_{n \geq 1} \in \oplus_{1}^{\infty} L^{2}(\mathbb{R})\right\} \\
M\left(f^{n}\right)_{n \geq 1}=\left(\lambda_{n} f^{n}\right)_{n \geq 1}
\end{array}\right.
$$

Plus précisément, notons $F: H \longrightarrow \oplus_{1}^{\infty} L^{2}(\mathbb{R})$, la transformation unitaire définie par $F \mathbf{u}=\left(f^{n}\right)_{n \geq 1}$, où, pour tout $n \geq 1$,

$$
f^{n}(p)=(2 \pi)^{-1 / 2} \text { l.i.m. } \int_{\Omega} \sum_{i=1}^{2} u_{i}\left(x_{1}, y\right) \overline{W_{n}^{i}(p, y)} \mathrm{e}^{-i p x_{1}} \underline{\rho} \mathrm{d} \mathbf{x}, \quad \text { p.p. } p \in \mathbb{R},
$$

avec $W_{n}(p)=\left(W_{n}^{1}(p, \cdot), W_{n}^{2}(p, \cdot)\right)$. On a $A=F^{-1} M F$. Ceci montre que l'étude de $A$ est intimement liée à celle des fonctions $\lambda_{n}, n \geq 1$.

Un procédé habituel dans l'étude spectrale, dit principe d'absorption limite, consiste à établir un prolongement par continuité, à l'axe réel, de la résolvante $z \longrightarrow R_{A}(z)=\left(A-z I_{d}\right)^{-1}$ définie a priori pour $z \in \mathbb{C}^{ \pm}$, pour une certaine topologie. Étant donnés $\mathbf{u}, \mathbf{v} \in H, z \in \mathbb{C}^{ \pm}$, et $\mathbf{f}=\left(f^{n}\right)_{n \geq 1}=F \mathbf{u}, \mathbf{g}=\left(g^{n}\right)_{n \geq 1}=F \mathbf{v}$, on a d'après ce qui précède

$$
\left\langle R_{A}(z) \mathbf{u}, \mathbf{v}\right\rangle_{H}=\sum_{n=1}^{\infty} \int_{\mathbb{R}} \frac{f^{n}(p) \overline{g^{n}(p)}}{\lambda_{n}(p)-z} \mathrm{~d} p
$$

et on montre qu'il suffit d'établir un prolongement par continuité pour $z \longrightarrow\left\langle R_{A}(z) \mathbf{u}, \mathbf{v}\right\rangle_{H}$. Dans [4] il est montré que les fonctions $\lambda_{n}$ tendent uniformément vers l'infini lorsque $n$ tend vers l'infini, ce qui permet de considérer séparément chacune des intégrales

$$
\int_{\mathbb{R}} \frac{f^{n}(p) \overline{g^{n}(p)}}{\lambda_{n}(p)-z} \mathrm{~d} p
$$

Les valeurs qui posent des problèmes pour une telle intégrale lorsque $z$ s'approche de l'axe réel sont les valeurs critiques de la fonction $\lambda_{n}$ (le cas où on est en dehors de ces valeurs critiques est facilement surmontable 
si le numérateur est höldérien). Aussi nous introduisons l'ensemble

$$
\Gamma=\left\{\lambda_{0} \in \mathbb{R} ; \exists p_{0} \in \mathbb{R} \text { et } n \geq 1 \text { tels que } \lambda_{0}=\lambda_{n}\left(p_{0}\right) \text { et } \lambda_{n}^{\prime}\left(p_{0}\right)=0\right\}
$$

des valeurs critiques des fonctions $\lambda_{n}$ que nous appelons seuils. Notons que ces dernières ne doivent pas être confondues avec les fréquences d'apparition de modes guidés appelées aussi seuils ou fréquences de coupure (cf. [2]).

Étant donné un seuil $\lambda_{0}=\lambda_{n}\left(p_{0}\right)$ avec $\lambda_{n}^{\prime}\left(p_{0}\right)=0$ pour un certain $p_{0}$ (supposons que $p_{0}$ est l'unique réel ainsi), nous introduisons l'ordre $N \geq 2$ de la racine $p_{0}$ de $p \longrightarrow \lambda_{n}(p)-\lambda_{n}\left(p_{0}\right)$. Si les dérivées de $f$ jusqu'à l'ordre $N-2$ s'annulent en $p_{0}$, alors le prolongement par continuité en $\lambda_{0}$ de l'intégrale ci-dessus ne pose plus de problème.

En acoustique (i.e. l'opérateur donné dans (2) avec des conditions aux bord en $y=0$ et $y=L$ ) les courbes de dispersion associées ont des propriétés simples. Elle sont symétriques et croissantes sur $\mathbb{R}_{+}$, leur dérivées ne s'annulent donc qu'en zéro. Par ailleurs, les dérivés secondes ne s'annulent pas $(c f$. $[4,7])$, i.e. l'ordre $N$ est toujours égal à 2 .

En élasticité il est donc naturel, d'une part, de se poser la question de la non monotonie des fonctions $\lambda_{n}$ sur $\mathbb{R}_{+}$et $\mathbb{R}_{-}$, et, d'autre part, de se demander si en un $p_{0}$ tel que $\lambda_{n}^{\prime}\left(p_{0}\right)=0$ on peut aussi avoir $\lambda_{n}^{\prime \prime}\left(p_{0}\right)=0, i . e$. l'ordre $N$ est-il supérieur ou égal à 3 ?

Le premier objectif de cet article est d'apporter une réponse à ces questions. On a les deux théorèmes suivants prouvés dans la section 3 :

Théorème 1.1. Les fonctions $\lambda_{n}, n \geq 1$, ne sont pas systématiquement monotones sur $\mathbb{R}_{+}$et $\mathbb{R}_{-}$.

Comme il a été dit plus haut, le problème de la non monotonie était un problème ouvert pour des opérateurs classiques. Nous lui apportons une réponse pour la première fois.

Théorème 1.2. Il existe des jeux de coefficients $\underline{\rho}, \underline{\lambda}, \underline{\mu}$ et des entiers $n$ pour lesquels on a $\lambda_{n}^{\prime}(0)=\lambda_{n}^{\prime \prime}(0)=$ $\lambda_{n}^{\prime \prime \prime}(0)=0$.

Contrairement au cas acoustique les fonctions $\lambda_{n}$ ne sont pas toutes symétriques mais satisfont une propriété de "symétrie globale" : pour tout $n$, il existe $n^{\prime}$ tel que $\lambda_{n^{\prime}}(-p)=\lambda_{n}(p)$ pour tout $p$. Pour montrer cela (voir [4]) on remarque que les opérateurs $A_{p}$ et $A_{-p}$ sont unitairement équivalents. Soient $p_{0} \in \mathbb{R}$ et $\lambda_{0}$ une valeur propre de l'opérateur $A_{p_{0}}$ de multiplicité $k$ (nous verrons que $k$ est soit égal à 1 soit à 2). Un résultat général de [9] dit qu'il existe $k$ fonctions (et pas plus), $\lambda_{n_{i}}, i=1, \ldots, k$, vérifiant $\lambda_{n_{i}}\left(p_{0}\right)=\lambda_{0}$. Concrètement on a les deux cas suivants selon que $\lambda_{n}(0)$ est une valeur propre simple ou double de $A_{0}$.

- si $\lambda_{n}(0)$ est simple alors $\lambda_{n}$ est une fonction symétrique,

- si $\lambda_{n}(0)$ est double alors les deux fonctions $\lambda_{n}$ et $\lambda_{n^{\prime}}$ telles que $\lambda_{n}(0)=\lambda_{n^{\prime}}(0)$ sont ou bien symétriques toutes les deux, ou bien symétriques l'une de l'autre.

C'est ce dernier cas qui illustre la non symétrie systématique à la différence de l'acoustique. On montre en effet explicitement, dans le cas de la bande homogène, qu'on peut avoir $\lambda_{n}^{\prime}(0) \neq 0$ ce qui implique que $\lambda_{n}$ et $\lambda_{n^{\prime}}$ ne sont pas symétriques (mais symétriques l'une de l'autre).

C'est de cette manière aussi qu'on démontrera le premier théorème ci-dessus. En effet, comme $\lambda_{n}^{\prime}(0) \neq 0$ et $\lambda_{n}^{\prime}(0)=-\lambda_{n^{\prime}}^{\prime}(0)$, l'une des fonctions $\lambda_{n}$ et $\lambda_{n^{\prime}}$ n'est pas monotone sur $\mathbb{R}_{+}$puisque chaque fonction $\lambda_{n}$, tend vers $+\infty$ lorsque $p$ tend vers $\pm \infty$. Ceci découle (voir [4]) de l'existence d'une constante $c_{R_{-}}>0$ telle que $\lambda_{n}(p) \geq c_{R_{-}}^{2} p^{2}$ pour tous $n$ et $p$.

La démonstration du théorème 1.2 ci-dessus utilise aussi des calculs explicites dans le cas de la bande homogène. On trouve d'abord l'expression exacte d'une relation de dispersion qui est une équation implicite de la forme $D(p, \lambda)=0$, où $D$ est une fonction de deux variables, exprimant une condition nécessaire et suffisante pour que $\lambda$ (à ne pas confondre avec le coefficient de Lamé $\underline{\lambda}$ qui, lui, est souligné) soit une valeur propre de l'opérateur réduit $A_{p}$. Ainsi, pour tous $n$ et $p$, on a $D\left(p, \lambda_{n}(p)\right)=0$. 
Le deuxième objectif de cet article est de présenter dans un cadre général la construction d'une relation de dispersion $D(p, \lambda)=0$, satisfaisant la propriété énoncée dans le

Théorème 1.3. La multiplicité de $\lambda$ comme racine de $D(p, \cdot)=0, p \in \mathbb{R}$, ou comme valeur propre de $A_{p}$ est la même.

Ce dernier résultat sera utilisé dans la démonstration du théorème 1.2 .

Une conséquence immédiate de ce théorème est que $\partial_{\lambda} D\left(p_{0}, \lambda_{0}\right) \neq 0$ si $\lambda_{0}$ est une valeur propre simple de $A_{p_{0}}$. Ainsi, pour une fonction $\lambda_{n}$ qui n'a aucun point commun avec les autres fonctions $\lambda_{n^{\prime}}$ on a $\partial_{\lambda} D\left(p, \lambda_{n}(p)\right) \neq 0$ pour tout $p$.

Une démonstration de ce résultat a été présentée par Skalak [12] et reprise dans [1], p. 340. Le raisonnement utilisé est grosso modo équivalent au suivant : soit $\lambda_{0}$ une valeur propre double de $A_{p_{0}}$, il existe alors $\lambda_{n_{1}}$ et $\lambda_{n_{2}}$ deux courbes (qu'on suppose distinctes) telles que $\lambda_{0}=\lambda_{n_{1}}\left(p_{0}\right)=\lambda_{n_{2}}\left(p_{0}\right)$. Le théorème des fonctions implicites dit alors que $\partial_{\lambda} D\left(p_{0}, \lambda_{0}\right)=0$. Inversement, supposons que $\partial_{\lambda} D\left(p_{0}, \lambda_{0}\right)=0$. Comme

$$
\partial_{p} D\left(p_{0}, \lambda_{0}\right)=-\lambda_{n_{1}}^{\prime}\left(p_{0}\right) \partial_{\lambda} D\left(p_{0}, \lambda_{0}\right)=0
$$

on a alors $\partial_{p} D\left(p_{0}, \lambda_{0}\right)=0$, et le point $\left(p_{0}, \lambda_{0}\right)$ est donc un point critique de la fonction $D(\cdot, \cdot)$. Si on suppose que ce dernier n'est pas dégénéré (i.e. si le Hessien en ce point est une matrice inversible), alors en appliquant la théorie de Morse ( $c f$. [10]) on montre qu'il existe une autre courbe $\lambda_{n_{2}}$, différente de $\lambda_{n_{1}}$, qui la coupe lorsque $p=p_{0}$. Forcément $\lambda_{0}$ est une valeur propre double de $A_{p_{0}}$.

La démonstration donnée est donc incomplète puisqu'elle ne tient pas compte du cas où $\left(p_{0}, \lambda_{0}\right)$ est un point dégénéré. Nous proposons une autre démonstration basée sur l'utilisation de l'argument spectral suivant : la résolvante d'un opérateur auto-adjoint admet un pôle de multiplicité 1 au voisinage d'une valeur propre isolée (voir la formule (3.21) dans [9], Chap. V, Sect. 3).

Pour une étude plus détaillée de la bande élastique et des courbes de dispersion nous renvoyons à [5].

Cet article est organisé comme suit.

Dans la section 2 nous construisons une relation de dispersion $D(p, \lambda)=0$ dans un cadre général et nous montrons le théorème 1.3. Comme applications nous donnerons la caractérisation des seuils et de leurs ordres à l'aide de $D$, et nous proposerons une nouvelle méthode numérique pour le calcul des courbes de dispersion et des seuils. Dans la section 3 nous calculons explicitement la fonction $D$ dans le cas homogène et nous démontrons les théorèmes 1.1 et 1.2 .

\section{RELATION DE DISPERSION : PROPRIÉTÉS ET CONSÉQUENCES}

Nous commencerons par construire la fonction $D$, définissant la relation de dispersion, et par donner ses propriétés. Nous démontrerons ensuite le théorème 1.3 .

\subsection{Multiplicité des valeurs propres des opérateurs réduits et construction de la relation de dispersion}

Étant donnés $p, \lambda \in \mathbb{C}$, cherchons les fonctions $\mathbf{u}=\left(u_{1}, u_{2}\right) \in D\left(A_{p}\right)$ telles que

$$
A_{p} \mathbf{u}-\lambda \mathbf{u}=0
$$

De telles fonctions $\mathbf{u}$ sont telles qu'on ait

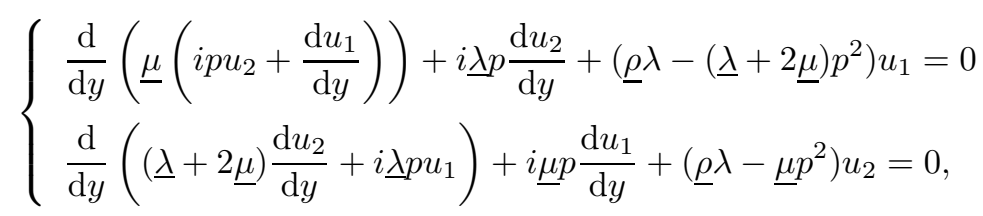


avec les conditions aux limites suivantes :

$$
\begin{aligned}
u_{1 \mid y=L} & =0, \quad u_{2 \mid y=L}=0 \\
\underline{\mu}\left(i p u_{2}+\frac{\mathrm{d} u_{1}}{\mathrm{~d} y}\right)_{\mid y=0} & =0, \quad\left((\underline{\lambda}+2 \underline{\mu}) \frac{\mathrm{d} u_{2}}{\mathrm{~d} y}+i \underline{\lambda} p u_{1}\right)_{\mid y=0}=0 .
\end{aligned}
$$

On ramène le système différentiel ci-dessus à un système du premier ordre en posant $v_{1}=u_{1}, v_{2}=u_{2}, v_{3}=$ $\underline{\mu}\left(i p u_{2}+\frac{\mathrm{d} u_{1}}{\mathrm{~d} y}\right)$ et $v_{4}=(\underline{\lambda}+2 \underline{\mu}) \frac{\mathrm{d} u_{2}}{\mathrm{~d} y}+i \underline{\lambda} p u_{1}$, et on obtient

$$
\begin{aligned}
\frac{\mathrm{d} v_{1}}{\mathrm{~d} y} & =\frac{v_{3}}{\underline{\mu}}-i p v_{2} \\
\frac{\mathrm{d} v_{2}}{\mathrm{~d} y} & =\frac{v_{4}}{\underline{\lambda}+2 \underline{\mu}}-\frac{i \underline{\lambda} p}{\underline{\lambda}+2 \underline{\mu}} v_{1} \\
\frac{\mathrm{d} v_{3}}{\mathrm{~d} y} & =\frac{-i \underline{\lambda} p}{\underline{\lambda}+2 \underline{\mu}} v_{4}-\left(\frac{\underline{\lambda}^{2} p^{2}}{\underline{\lambda}+2 \underline{\mu}}+\underline{\rho} \lambda-(\underline{\lambda}+2 \underline{\mu}) p^{2}\right) v_{1} \\
\frac{\mathrm{d} v_{4}}{\mathrm{~d} y} & =-i p v_{3}-\underline{\rho} \lambda v_{2},
\end{aligned}
$$

qui se met alors sous la forme

$$
\frac{\mathrm{d} V}{\mathrm{~d} y}=M(y, p, \lambda) V
$$

où $V=\left(v_{1}, v_{2}, v_{3}, v_{4}\right)$ et

$$
M(y, p, \lambda)=\left(\begin{array}{cccc}
0 & -i p & \frac{1}{\mu} & 0 \\
-\frac{i \underline{\lambda} p}{\underline{\lambda}+2 \underline{\mu}} & 0 & 0 & \frac{1}{\underline{\lambda}+2 \underline{\mu}} \\
-\left(\frac{\lambda^{2} p^{2}}{\underline{\lambda}+2 \underline{\mu}}+\underline{\rho} \lambda-(\underline{\lambda}+2 \underline{\mu}) p^{2}\right) & 0 & 0 & \frac{-i \underline{p} p}{\underline{\lambda}+2 \underline{\mu}} \\
0 & -\underline{\rho} \lambda & -i p & 0
\end{array}\right) .
$$

Les conditions aux bords se traduisent par :

$$
\begin{aligned}
& v_{1}(L)=0, \quad v_{2}(L)=0, \\
& v_{3}(0)=0, \quad v_{4}(0)=0 .
\end{aligned}
$$

Les solutions du système homogène (6) sont données grâce à la résolvante $\mathcal{R}(y, s, p, \lambda)$ du système. C'est une fonction de $y$ et $s$ à valeurs matricielles. Elle satisfait (6) pour tout $s$ fixé avec la condition $\mathcal{R}(s, s, p, \lambda)=I_{d}$. Ainsi, pour tout $\alpha=\left(\alpha_{1}^{\prime}, \alpha_{2}^{\prime}, \alpha_{1}, \alpha_{2}\right)$, la solution $V$ de (6) qui prend la valeur $\alpha$ en $y=L$ (on choisit $s=L$ ) est donnée par $V(y)=\mathcal{R}(y, L, p, \lambda) \alpha$. Immédiatement, en prenant (8) en compte il vient $\alpha_{1}^{\prime}=\alpha_{2}^{\prime}=0$.

Introduisons les matrices $\left(R_{i j}(y, s, p, \lambda)\right)$ et $\left(D_{i j}(y, s, p, \lambda)\right)$, définies par

$$
R_{i j}(y, s, p, \lambda)=\mathcal{R}_{i, j+2}(y, s, p, z), \quad D_{i j}(y, s, p, \lambda)=\mathcal{R}_{i+2, j+2}(y, s, p, z), \quad 1 \leq i, j \leq 2 .
$$

La matrice $\mathcal{R}$ s'écrit alors :

$$
\mathcal{R}=\left(\begin{array}{ll}
\cdot & R_{i j} \\
\cdot & D_{i j}
\end{array}\right),
$$


d'où $\left(v_{3}, v_{4}\right)(y)=\left(D_{i j}(y, L, p, \lambda)\right)\left(\alpha_{1}, \alpha_{2}\right)$, et la condition (9) se réécrit

$$
\left(D_{i j}(0, L, p, \lambda)\right)\left(\alpha_{1}, \alpha_{2}\right)=0
$$

Il est alors clair que les fonctions $\mathbf{u}$ satisfaisant (3) sont données par

$$
\mathbf{u}(y)=\left(R_{i j}(y, L, p, \lambda)\right)\left(\alpha_{1}, \alpha_{2}\right)
$$

où $\left(\alpha_{1}, \alpha_{2}\right)$ satisfait (11). Une condition nécessaire et suffisante pour que $\lambda$ soit une valeur propre de $A_{p}$ est donc

$$
D(p, \lambda):=\operatorname{det}\left(D_{i j}(0, L, p, \lambda)\right)=0 \text {. }
$$

Par ailleurs, l'espace vectoriel des fonctions données par (12) est de dimension au plus égale à 2 . Pour $p$ réel (cas dans lequel $A_{p}$ est auto-adjoint) la multiplicité des valeurs propres des opérateurs réduits est donc au plus égale à 2. Plus précisément, on a les trois possibilités suivantes :

1. La matrice $\left(D_{i j}(0, L, p, \lambda)\right)$ est inversible. Dans ce cas $\lambda$ n'est pas valeur propre de $A_{p}$.

2. La matrice $\left(D_{i j}(0, L, p, \lambda)\right)$ n'est pas inversible et n'est pas nulle. Dans ce cas $\lambda$ est une valeur propre simple de $A_{p}$.

3. La matrice $\left(D_{i j}(0, L, p, \lambda)\right)$ est nulle. Dans ce cas $\lambda$ est une valeur propre double de $A_{p}$.

Dans le système homogène (6), pour tout $y$ fixé, la matrice $M(y, p, \lambda)$ dépend analytiquement des variables complexes $p$ et $\lambda$. Il est connu (cf. [6]) que ceci entraîne aussi l'analyticité, pour tous $y$ et $s$ fixés, de la résolvante $\mathcal{R}(y, s, \cdot, \cdot)$. par rapport à $p$ et $\lambda$. Il en est de même pour les matrices $\left(D_{i j}(y, s, p, z)\right)$ et $\left(R_{i j}(y, s, p, z)\right)$ et, par conséquent, la fonction $D$ est analytique.

Nous allons voir maintenant que $D$ est symétrique par rapport à $p$. Il suffit pour cela de montrer que les fonctions $D_{11}(0, L, \cdot, \lambda)$ et $D_{22}(0, L, \cdot, \lambda)$ sont symétriques, et que $D_{12}(0, L, \cdot, \lambda)$ et $D_{21}(0, L, \cdot, \lambda)$ sont antisymétriques.

En effet, pour tout $p$, le vecteur $V_{p}=\left(v_{p}^{1}, v_{p}^{2}, v_{p}^{3}, v_{p}^{4}\right)$ tel que $v_{p}^{i}=\mathcal{R}_{i, 3}(\cdot, L, p, \lambda), i=1, \ldots, 4$, est l'unique solution de $\frac{\mathrm{d} V}{\mathrm{~d} y}=M(y, p, \lambda) V$, avec la condition $V(L)=(0,0,1,0)$. Par ailleurs, on vérifie que $W_{p}=$ $\left(v_{p}^{1},-v_{p}^{2}, v_{p}^{3},-v_{p}^{4}\right)$ est une solution de $\frac{\mathrm{d} V}{\mathrm{~d} y}=M(y,-p, \lambda) V$ vérifiant la condition $V(L)=(0,0,1,0)$, d'où $W_{p}=V_{-p}$. Comme $v_{p}^{3}=D_{11}(y, L, p, \lambda)$ et $v_{p}^{4}=D_{21}(y, L, p, \lambda)$, on déduit que $D_{11}(0, L, p, \lambda)$ est une fonction paire en $p$ et que $D_{21}(0, L, p, \lambda)$ est impaire. De la même manière, on montre que $D_{22}(0, L, \cdot, \lambda)$ est paire et que $D_{12}(0, L, \cdot, \lambda)$ est impaire.

\subsection{Démonstration $\mathbf{d u}$ théorème $\mathbf{1 . 3}$}

Nous allons tout d'abord chercher une expression de la résolvante $\left(A_{p}-z I_{d}\right)^{-1}$ en fonction de $D$. Nous procéderons ensuite en deux étapes pour la démonstration.

Soient $z \in \mathbb{C}$ qui ne soit pas une valeur propre de $A_{p}$, et $\mathbf{f}=\left(f_{1}, f_{2}\right) \in \bar{H}$. On vérifie que la fonction $\mathbf{u}=\left(A_{p}-z I_{d}\right)^{-1} \mathbf{f}$ est égale à $\left(v_{1}, v_{2}\right)$, où $V=\left(v_{1}, v_{2}, v_{3}, v_{4}\right)$ est la solution du système inhomogène

$$
\frac{\mathrm{d} V}{\mathrm{~d} y}=M(y, p, z) V+\mathbf{f}^{\prime}
$$

dans lequel $\mathbf{f}^{\prime}=-\underline{\rho}\left(0,0, f_{1}, f_{2}\right)$, avec les conditions aux limites $(8,9)$.

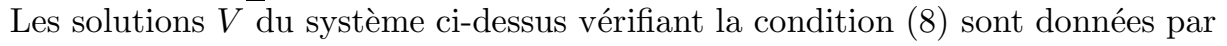

$$
-\int_{y}^{L} \mathcal{R}(y, s, p, z) \mathbf{f}^{\prime}(s) \mathrm{d} s+\mathcal{R}(y, L, p, z) \alpha^{\prime}
$$


où $\alpha^{\prime}=\left(0,0, \alpha_{1}, \alpha_{2}\right)$ et $\mathcal{R}(y, s, p, z)$ est la résolvante du système homogène $(6)$.

Prenant en compte (10) on voit que $V$ vérifie (9), i.e. $v_{3}(0)=v_{4}(0)=0$, si et seulement si $\alpha=\left(\alpha_{1}, \alpha_{2}\right)$ satisfait

$$
\int_{0}^{L}\left(D_{i j}(0, s, p, z)\right) \underline{\rho} \mathbf{f}(s) \mathrm{d} s+\left(D_{i j}(0, L, p, z)\right) \alpha=0 .
$$

Puisque $\left(D_{i j}(0, L, p, z)\right)$ est inversible (car $z$ n'est pas une valeur propre de $\left.A_{p}\right)$, cette relation équivaut à

$$
\alpha=-\left(D_{i j}(0, L, p, z)\right)^{-1} \int_{0}^{L}\left(D_{i j}(0, s, p, z)\right) \underline{\rho} \mathbf{f}(s) \mathrm{d} s .
$$

La résolvante de l'opérateur $A_{p}$ est donc donnée par :

$$
\begin{aligned}
\left(A_{p}-z I_{d}\right)^{-1} \mathbf{f}= & \int_{y}^{L}\left(R_{i j}(y, s, p, z)\right) \underline{\rho} \mathbf{f}(s) \mathrm{d} s \\
& -\left(R_{i j}(y, L, p, z)\right)\left(D_{i j}(0, L, p, z)\right)^{-1} \int_{0}^{L}\left(D_{i j}(0, s, p, z)\right) \underline{\rho} \mathbf{f}(s) \mathrm{d} s .
\end{aligned}
$$

Les pôles de $z \longrightarrow\left(A_{p}-z I_{d}\right)^{-1} \in B(\bar{H})$, et leur multiplicité, sont ceux de $z \longrightarrow R_{1}(z) R_{2}(z) R_{3}(z)$, où

$$
\begin{aligned}
& R_{1}(z) \alpha=\left(R_{i j}(y, L, p, z)\right) \alpha, \quad \forall \alpha \in \mathbb{C}^{2}, \quad R_{2}(z)=\left(D_{i j}(0, L, p, z)\right)^{-1} \\
& R_{3}(z) \mathbf{f}=\int_{0}^{L}\left(D_{i j}(0, s, p, z)\right) \underline{\mathbf{f}}(s) \mathrm{d} s, \quad \forall \mathbf{f} \in \bar{H} .
\end{aligned}
$$

Nous aurons besoin du lemme suivant :

Lemme 2.1. Soient deux fonctions holomorphes $z \longrightarrow R_{3}(z) \in B\left(\bar{H}, \mathbb{C}^{2}\right)$ et $z \longrightarrow R_{1}(z) \in B\left(\mathbb{C}^{2}, \bar{H}\right)$ telles que, pour un certain $z_{0} \in \mathbb{C}, R_{3}\left(z_{0}\right)$ soit un opérateur surjectif et $R_{1}\left(z_{0}\right)$ un opérateur injectif.

Si $z \longrightarrow R_{2}(z) \in B\left(\mathbb{C}^{2}\right)$ est une fonction méromorphe, alors la fonction méromorphe

$$
z \longmapsto R_{0}(z)=R_{1}(z) R_{2}(z) R_{3}(z) \in B(\bar{H}),
$$

a les même pôles, avec les mêmes multiplicités.

Démonstration. Soit $z_{0}$ un pôle d'ordre $m$ de $R_{2}$, montrons qu'il est aussi pôle de même ordre pour $R_{0}(z)$.

Pour $z$ appartenant à un voisinage de $z_{0}$, on a

$$
\left\|\left(z-z_{0}\right)^{m} R_{0}(z)\right\|_{B(\bar{H})} \leq\left\|R_{1}(z)\right\|_{B\left(\mathbb{C}^{2}, \bar{H}\right)}\left\|\left(z-z_{0}\right)^{m} R_{2}(z)\right\|_{B\left(\mathbb{C}^{2}\right)}\left\|R_{3}(z)\right\|_{B\left(\bar{H}, \mathbb{C}^{2}\right)} \leq c_{0},
$$

où $c_{0}$ est une constante indépendante de $z$, ce qui montre que $z_{0}$ est un pôle de $R_{0}$ d'ordre au plus égal à $m$.

Inversement, montrons que $z_{0}$ est un pôle de $R_{0}$ d'ordre au moins égal à $m$. Sachant que $z_{0}$ est un pôle d'ordre $m$ de $R_{2}$, il existe $\alpha_{0} \in \mathbb{C}^{2}$ tel que

$$
\left|z-z_{0}\right|^{m}|| R_{2}(z) \alpha_{0} \|_{\mathbb{C}^{2}} \geq 2 c_{1}>0
$$

pour tout $z$ dans un voisinage de $z_{0}$. Comme l'opérateur $R_{3}\left(z_{0}\right)$ est surjectif, il existe $\mathbf{f}_{0} \in \bar{H}$ telle que $\alpha_{0}=R_{3}\left(z_{0}\right) \mathbf{f}_{0}$. Posons $\alpha_{z}=R_{3}(z) \mathbf{f}_{0}$. Lorsque $z \rightarrow z_{0}$ on a $\alpha_{z} \rightarrow \alpha_{0}$, lorsque $z \rightarrow z_{0}$. D'après (16), on a alors $\left|z-z_{0}\right|^{m}|| R_{2}(z) \alpha_{z} \|_{\mathbb{C}^{2}} \geq c_{1}>0$, pour tout $z$ dans un voisinage de $z_{0}$. 
Par ailleurs, comme $R_{1}\left(z_{0}\right)$ est injectif, on vérifie que pour tout $z$ dans un voisinage de $z_{0}$ et tout $\alpha \in \mathbb{C}^{2}$, on a $\|\left. R_{1}(z) \alpha\right|_{\bar{H}} \geq c|\alpha|_{\mathbb{C}^{2}}$, où $c$ est une constante indépendante de $z$ et de $\alpha$. On a aussi

$$
\left|z-z_{0}\right|^{m}|| R_{0}(z) \mathbf{f}_{0}\left\|_{\bar{H}} \geq c\left|z-z_{0}\right|^{m}|| R_{2}(z) \alpha_{z}\right\| \geq c c_{1}
$$

et par conséquent $\left|z-z_{0}\right|^{m}\left\|R_{0}(z)\right\|_{B(\bar{H})} \geq c c_{1} /\left\|\mathbf{f}_{0}\right\|_{\bar{H}}$ pour tout $z$ appartenant à un voisinage de $z_{0}$. Ceci montre que $z_{0}$ est un pôle de $R_{0}$ d'ordre au moins $m$.

Première étape de la démonstration du théorème 1.3.

Montrons que les pôles de la résolvante $\left(A_{p}-z I_{d}\right)^{-1}$, et leur multiplicité, sont ceux de $z \longrightarrow\left(D_{i j}(0, L, p, z)\right)^{-1}$. Il suffit pour cela d'appliquer le lemme précédent et de montrer que $R_{1}(z), R_{2}(z)$ et $R_{3}(z)$, définis par $(14,15)$, satisfont les hypothèses de ce dernier.

Il est clair que $R_{2}$ est méromorphe [cf. (18)] et que $R_{1}$ et $R_{3}$ sont holomorphes puisque les solutions du système homogène (6) dépendent analytiquement de $z$.

Raisonnons par l'absurde et supposons que $R_{3}(z)$ ne soit pas surjectif, il existe alors une matrice non nulle $M^{\prime}$ telle que

$$
M^{\prime} \int_{0}^{L}\left(D_{i j}(0, s, p, z)\right) \underline{\rho} \mathbf{f}(s) \mathrm{d} s=\int_{0}^{L} M^{\prime}\left(D_{i j}(0, s, p, z)\right) \underline{\rho} \mathbf{f}(s) \mathrm{d} s=0, \quad \forall \mathbf{f} \in \bar{H}
$$

ce qui donne $M^{\prime}\left(D_{i j}(0, s, p, z)\right)=0$, pour tout $s \in[0, L]$, et contredit le fait que $\left(D_{i j}(0,0, p, z)\right)$ est la matrice identité.

Pour vérifier que $R_{1}(z)$ est injectif il suffit de montrer qu'il existe $y \in[0, L]$ tel que la matrice $\left(R_{i j}(y, L, p, z)\right)$ soit inversible. Raisonnons par l'absurde encore une fois et supposons que

$$
R_{11}(y, L, p, z) R_{22}(y, L, p, z)-R_{21}(y, L, p, z) R_{12}(y, L, p, z)=0, \quad \forall y \in[0, L]
$$

Rappelons que, pour $j=1,2,\left(R_{1 j}(\cdot, L, p, z), R_{2 j}(\cdot, L, p, z), D_{1 j}(\cdot, L, p, z), D_{2 j}(\cdot, L, p, z)\right)$ est la solution du système (6) qui prend la valeur $\left(0,0, \delta_{1 j}, \delta_{2 j}\right)$ en $y=L$. On a donc

$$
\left\{\begin{array}{l}
\frac{\mathrm{d}}{\mathrm{d} y} R_{1 j}(y, L, p, z)=\frac{D_{1 j}(y, L, p, z)}{\mu}-i p R_{2 j}(y, L, p, z), \\
\frac{\mathrm{d}}{\mathrm{d} y} R_{2 j}(y, L, p, z)=\frac{D_{2 j}(y, L, p, z)}{\underline{\lambda}+2 \underline{\mu}}-\frac{i \underline{\lambda} p}{\underline{\lambda}+2 \underline{\mu}} R_{1 j}(y, L, p, z) .
\end{array}\right.
$$

En prenant l'intégrale entre $y$ et $L$ dans les deux expressions ci-dessus, on vérifie que

$$
\lim _{y \rightarrow L} \frac{R_{12}(y, L, p, z)}{L-y}=\lim _{y \rightarrow L} \frac{R_{21}(y, L, p, z)}{L-y}=0
$$

et que

$$
\liminf _{y \rightarrow L} \frac{\left|R_{j j}(y, L, p, z)\right|}{L-y} \geq \frac{1}{\|\underline{\lambda}+2 \underline{\mu}\|_{\infty}}, \quad \text { pour } j=1,2
$$

En divisant par $(L-y)^{2}$ dans $(17)$ et en passant à la limite lorsque $y$ tend vers $L$, on aboutit à une contradiction. 
Deuxième étape et fin de la démonstration

Une valeur propre $\lambda$ de $A_{p}$ étant un pôle simple pour la résolvante $z \longrightarrow\left(A_{p}-z I_{d}\right)^{-1}$, d'après l'étape précédente elle est aussi un pôle simple pour

$$
z \longrightarrow\left(D_{i j}(0, L, p, z)\right)^{-1}=\frac{\left(D_{i j}^{\dagger}(0, L, p, z)\right)}{D(p, z)}
$$

où $\left(D_{i j}^{\dagger}(0, L, p, z)\right)$ est la matrice transposée de la matrice des cofacteurs.

Si $\lambda$ est une valeur propre simple alors il existe $i$ et $j$ tels que $D_{i j}^{\dagger}(0, L, p, \lambda) \neq 0$. Comme $z=\lambda$ est un pôle simple pour

$$
z \longmapsto \frac{D_{i j}^{\dagger}(0, L, p, z)}{D(p, z)}
$$

alors $z=\lambda$ est forcément une racine simple pour $D(p, z)$, i.e. $\partial_{\lambda} D(p, \lambda) \neq 0$.

Inversement, si $\lambda$ n'est pas une valeur propre simple (dans ce cas c'est une valeur propre double) on a vu que c'est une racine de toutes les fonctions $D_{i j}(0, L, p, \cdot), 1 \leq i, j \leq 2$, et c'est donc une racine, au moins double, de $D(p, \cdot)$, ce qui donne $\partial_{\lambda} D(p, \lambda)=0$. Montrons que dans ce cas

$$
\partial_{\lambda}^{2} D(p, \lambda) \neq 0
$$

Soit $m_{0}$ le plus grand entier tel que toutes les fonctions $D_{i j}(0, L, p, \cdot)$ aient $\lambda$ comme racine de multiplicité supérieure ou égale à $m_{0}$. Alors $\lambda$ est une racine de $D(p, \cdot)$ de multiplicité supérieure ou égale à $2 m_{0}$ et il existe $i$ et $j$ tels que $\lambda$ soit une racine de multiplicité $m_{0}$ de $D_{i j}(0, L, p, \cdot)$. Comme la fonction (18) a un pôle de degré au plus égal à 1 , on déduit que $m_{0} \leq 1$.

Raisonnons maintenant par l'absurde et supposons que (19) n'ait pas lieu, ce qui revient à dire que la fonction $D(p, \cdot)$ admet $\lambda$ comme une racine de multiplicité au moins égale à trois. D'après ce qui précède, il existe alors $i, j$ tels que $D_{i j}(0, L, p, \cdot)$ admette $\lambda$ comme une racine de multiplicité au plus 1 (car $\left.m_{0} \leq 1\right)$. Par conséquent, la fonction (18) admet un pôle de multiplicité au moins égale à deux, ce qui est une contradiction.

\subsection{Caractérisation des seuils à l'aide de la relation de dispersion}

Soit un seuil $\lambda_{0}=\lambda_{n}\left(p_{0}\right)$ avec $\lambda_{n}^{\prime}\left(p_{0}\right)=0$. Nous distinguons le cas où $\lambda_{0}$ est une valeur propre simple de $A_{p_{0}}$ de celui où elle est double.

Cas où $\lambda_{0}$ est une valeur propre simple

D'après le théorème 1.3 on a $D\left(p_{0}, \lambda_{0}\right)=0$ et $\partial_{\lambda} D\left(p_{0}, \lambda_{0}\right) \neq 0$. En dérivant par rapport à $p$ l'égalité $D\left(p, \lambda_{n}(p)\right)=0$, il vient

$$
\lambda_{n}^{\prime}(p)=-\frac{\partial_{p} D\left(p, \lambda_{n}(p)\right)}{\partial_{\lambda} D\left(p, \lambda_{n}(p)\right)},
$$

dans un voisinage de $p_{0}$. Ainsi $\lambda_{0}$ est un seuil s'il existe $p_{0} \in \mathbb{R}$ tel que $\partial_{p} D\left(p_{0}, \lambda_{0}\right)=0$.

Par ailleurs, si $N$ désigne l'ordre de la racine $p_{0}$ de la fonction $p \longrightarrow \lambda_{n}(p)-\lambda_{n}\left(p_{0}\right)$, on peut dériver $\lambda_{n}$ jusqu'à l'ordre $N$ en utilisant (20) et vérifier que la racine $p_{0}$ de $D\left(\cdot, \lambda_{0}\right)$ est d'ordre $N$ aussi.

On a vu plus haut que pour tout $\lambda$ la fonction $D(\cdot, \lambda)$ est symétrique, donc $\partial_{p} D(0, \lambda)=0$ et $(20)$ donne $\lambda_{n}^{\prime}(0)=0$. On retrouve ainsi un résultat déjà connu découlant de la symétrie de la fonction $\lambda_{n}$. 
Cas où $\lambda_{0}$ est une valeur propre double

Dans ce cas on a $D\left(p_{0}, \lambda_{0}\right)=0, \partial_{\lambda} D\left(p_{0}, \lambda_{0}\right)=0$ et $\partial_{\lambda}^{2} D\left(p_{0}, \lambda_{0}\right) \neq 0$, et il existe $n_{1} \geq 1$ et $n_{2} \geq 1$, avec $n_{1} \neq n_{2}$ et $\lambda_{n_{1}}\left(p_{0}\right)=\lambda_{n_{2}}\left(p_{0}\right)=\lambda_{0}$, tels que

$$
D\left(p, \lambda_{n_{1}}(p)\right)=D\left(p, \lambda_{n_{2}}(p)\right)=0 .
$$

En dérivant une fois l'une des deux fonctions ci-dessus et en utilisant $\partial_{\lambda} D\left(p_{0}, \lambda_{0}\right)=0$ il vient $\partial_{p} D\left(p_{0}, \lambda_{0}\right)=0$. En les dérivant deux fois, on vérifie que $\lambda_{n_{1}}^{\prime}\left(p_{0}\right)$ et $\lambda_{n_{2}}^{\prime}\left(p_{0}\right)$ sont solutions de l'équation du second degré (en $X$ ) suivante :

$$
\partial_{\lambda}^{2} D\left(p_{0}, \lambda_{0}\right) X^{2}+2 \partial_{\lambda p}^{2} D\left(p_{0}, \lambda_{0}\right) X+\partial_{p}^{2} D\left(p_{0}, \lambda_{0}\right)=0
$$

Ainsi, $\lambda_{0}$ est un seuil si l'une des solutions est nulle, i.e. $\partial_{p}^{2} D\left(p_{0}, \lambda_{0}\right)=0$.

Si $\partial_{\lambda p}^{2} D\left(p_{0}, \lambda_{0}\right) \neq 0$, ce qui équivaut à ce que l'une des solutions de l'équation ci-dessus soit non nulle, et en calculant toutes les dérivées dans (21) jusqu'à l'ordre $N+1$, où $N$ est l'ordre de la racine $p_{0}$ de $\lambda_{n_{1}}(p)-\lambda_{n_{1}}\left(p_{0}\right)$, on vérifie que $p_{0}$ est une racine d'ordre $N+1$ de $p \longrightarrow D\left(p, \lambda_{0}\right)-D\left(p_{0}, \lambda_{0}\right)$.

Pour $p_{0}=0$ on a

$$
\left\{\lambda_{n_{1}}^{\prime}(0), \lambda_{n_{2}}^{\prime}(0)\right\}=\left\{ \pm\left(\frac{-\partial_{p}^{2} D\left(0, \lambda_{0}\right)}{\partial_{\lambda}^{2} D\left(0, \lambda_{0}\right)}\right)^{1 / 2}\right\}
$$

Si $\partial_{p}^{2} D\left(0, \lambda_{0}\right)=0$, alors $\lambda_{0}$ est un seuil. Dans le cas contraire, $\lambda_{n_{1}}^{\prime}(0)=-\lambda_{n_{2}}(0)$ est non nul et l'une des fonctions $\lambda_{n_{1}}$ ou $\lambda_{n_{2}}$ n'est donc pas monotone sur $\mathbb{R}_{+}$. On a ainsi retrouvé autrement un résultat découlant de la symétrie, l'une par rapport à l'autre, des fonctions $\lambda_{n_{1}}$ et $\lambda_{n_{2}}$.

\subsection{Méthode numérique utilisant la relation de dispersion}

La méthode numérique habituelle pour le calcul des courbes de dispersion est basée sur la formulation variationnelle du problème spectral associé. En effet, pour tout $p \in \mathbb{R}$ fixé, $\lambda_{n}(p), n \geq 1$, sont les solutions du problème suivant :

$$
\exists \mathbf{u} \in \overline{\mathbf{V}}, \mathbf{u} \neq 0, \quad a_{p}(\mathbf{u}, \mathbf{v})=\lambda_{n}(p)\langle\mathbf{u}, \mathbf{v}\rangle_{\bar{H}}, \quad \forall \mathbf{v} \in \overline{\mathbf{V}}
$$

Ensuite une discrétisation, en éléments finis, de l'espace $\overline{\mathbf{V}}$ et une discrétisation de la forme hermitienne $a_{p}(\cdot, \cdot)$ sont utilisées pour obtenir un problème matriciel.

La méthode que nous proposons utilise la relation de dispersion $D(p, \lambda)=0$. Elle consiste dans une première étape à calculer numériquement la fonction $D$, ainsi que ses dérivées, puis à calculer ses racines. Son avantage par rapport à la première est qu'elle est plus économique en mémoire machine puisqu'elle ne nécessite pas le stockage de matrices. En complément nous présentons une méthode de calcul des courbes de dispersion, des seuils, des points de croisement entre les courbes de dispersion et des vecteurs propres des opérateurs réduits. Pour la mise en œuvre nous renvoyons à [5].

\subsubsection{Calcul des fonctions $D, \partial_{p} D$ et $\partial_{\lambda} D$}

Le réel $L$ est fixé. Rappelons que pour tous $p, \lambda \in \mathbb{R}$, les quantités $D_{i j}(0, L, p, \lambda), 1 \leq i, j \leq 2$, se caractérisent comme suit : si $V=\left(v_{1}, v_{2}, v_{3}, v_{4}\right)$ est la solution du système

$$
\frac{\mathrm{d} V}{\mathrm{~d} y}=M(y, p, \lambda) V,
$$

avec la condition $V(L)=(0,0,1,0)(\operatorname{resp} . \quad V(L)=(0,0,0,1))$ alors $D_{11}(0, L, p, \lambda)=v_{3}(0), D_{21}(0, L, p, \lambda)=$ $v_{4}(0)$ (resp. $\left.D_{12}(0, L, p, \lambda)=v_{3}(0), D_{22}(0, L, p, \lambda)=v_{4}(0)\right)$. Ceci permet de calculer $D(p, \lambda)$ grâce à $(13)$. 
La solution $V$ dépend analytiquement de $p$ et $\lambda$. En dérivant par rapport à $p$, il vient

$$
\frac{\mathrm{d} \partial_{p} V}{\mathrm{~d} y}=M(y, p, \lambda) \partial_{p} V+\partial_{p} M(y, p, \lambda) V
$$

avec la condition finale $\partial_{p} V(L, p, \lambda)=0$. De même, en dérivant par rapport $\lambda$, on a

$$
\frac{\mathrm{d} \partial_{\lambda} V}{\mathrm{~d} y}=M(y, p, \lambda) \partial_{\lambda} V+\partial_{\lambda} M(y, p, \lambda) V
$$

avec la condition finale $\partial_{\lambda} V(L, p, \lambda)=0$. Ceci permet d'obtenir $\partial_{p} D_{i j}(0, L, p, \lambda)\left(\operatorname{resp} . \partial_{\lambda} D_{i j}(0, L, p, \lambda)\right)$ et donc $\partial_{p} D(p, \lambda)\left(\operatorname{resp} . \partial_{\lambda} D(p, \lambda)\right)$.

Pour le calcul numérique de $D, \partial_{p} D$ et $\partial_{\lambda} D$ on utilise une méthode numérique pour résoudre les trois systèmes différentiels ci-dessus avec la condition finale correspondante en $y=L$. Par des considérations analogues, on calcule $\partial_{p}^{2} D\left(\operatorname{resp} . \partial_{p, \lambda}^{2} D, \partial_{\lambda}^{2} D\right)$.

En pratique nous avons utilisé l'algorithme de Runge-Kutta explicite d'ordre 4 pour la mise en œuvre, et, supposant que $\underline{\rho}, \underline{\lambda}$ et $\underline{\mu}$ sont de classe $C^{4}$, nous avons montré une estimation d'erreur d'ordre 4 sur le calcul numérique de $\bar{D}$ et de ses dérivées ( $c f .[5])$.

\subsubsection{Méthode de calcul des courbes de dispersion}

On utilise un algorithme de "prédiction-correction" pour calculer une courbe $\lambda_{n}$ sur un intervalle $(a, b)$, connaissant $\lambda_{n}(a)$.

Si, pour $p_{0} \in[a, b]$, la valeur propre $\lambda_{n}\left(p_{0}\right)$ est simple (ce qui équivaut aussi à ce que $\lambda_{n}$ ne croise pas une autre courbe pour ce $\left.p_{0}\right)$ alors $\partial_{\lambda} D\left(p_{0}, \lambda_{n}\left(p_{0}\right)\right) \neq 0$ et donc

$$
\frac{\mathrm{d} \lambda_{n}}{\mathrm{~d} p}\left(p_{0}\right)=-\frac{\partial_{p} D}{\partial_{\lambda} D}\left(p_{0}, \lambda_{n}\left(p_{0}\right)\right) .
$$

Pour $p_{1}$ assez proche de $p_{0}$, la première étape de l'algorithme de "prédiction-correction" consiste à approcher $\lambda_{n}\left(p_{1}\right)$ par

$$
\theta=\lambda_{n}\left(p_{0}\right)+\frac{\mathrm{d} \lambda_{n}}{\mathrm{~d} p}\left(p_{0}\right)\left(p_{1}-p_{0}\right)=\lambda_{n}\left(p_{0}\right)-\frac{\partial_{p} D}{\partial_{\lambda} D}\left(p_{0}, \lambda_{n}\left(p_{0}\right)\right)\left(p_{1}-p_{0}\right)
$$

Dans la deuxième étape on corrige cette approximation en appliquant l'algorithme de Newton à la fonction $D\left(p_{1}, \cdot\right)$ au point $\theta$.

Étant donnée une discrétisation $a=p_{0}<\ldots<p_{k_{b}}$ de $(a, b)$ et connaissant $\lambda_{n}(a)$, on calcule ainsi de proche en proche $\lambda_{n}\left(p_{k}\right)$ pour $k=1, \ldots, k_{b}$.

\subsubsection{Calcul des seuils}

Soit $a=p_{0}<\ldots<p_{k_{b}}, k=1, \ldots, k_{b}$, une discrétisation de $(a, b)$. Posons

$$
\theta_{k}^{\prime}:=\lambda_{n}^{\prime}\left(p_{k}\right)=-\frac{\partial_{p} D}{\partial_{\lambda} D}\left(p_{k}, \lambda_{n}\left(p_{k}\right)\right)
$$

Si pour un certain $k$ les signes de $\theta_{k-1}^{\prime}$ et $\theta_{k}^{\prime}$ sont opposés alors il existe $\tilde{p} \in\left(p_{k-1}, p_{k}\right)$ tel que $\lambda_{n}^{\prime}(\tilde{p})=0$. On peut alors utiliser la méthode de la sécante (ou celle de Newton) pour calculer la racine $\tilde{p}$ de $\lambda_{n}^{\prime}$. Le seuil correspondant est donc donné par $\tilde{\lambda}=\lambda_{n}(\tilde{p})$.

Étant donnée que $(\tilde{p}, \tilde{\lambda})$ est une racine de la fonction $(p, \lambda) \longrightarrow\left(D, \partial_{p} D\right)$, une autre méthode pour calculer $(\tilde{p}, \tilde{\lambda})$ consiste à appliquer la méthode de Newton à cette fonction. 
2.4.4. Calcul des points où se coupent deux courbes de dispersion

Deux courbes $\lambda_{n}$ et $\lambda_{n^{\prime}}$ se coupent en $\widehat{p}$, i.e. $\lambda_{n}(\widehat{p})=\lambda_{n^{\prime}}(\widehat{p})=\widehat{\lambda}$, si et seulement $\operatorname{si} \partial_{\lambda} D(\widehat{p}, \widehat{\lambda})=0$ avec $D(\widehat{p}, \widehat{\lambda})=0$.

Trouver $(\widehat{p}, \widehat{\lambda})$ revient donc à chercher les racines de la fonction $(p, \lambda) \longrightarrow\left(D, \partial_{\lambda} D\right)$.

Pour le calcul numérique de $(\widehat{p}, \widehat{\lambda})$ on applique la méthode de Newton à $(p, \lambda) \longrightarrow\left(D, \partial_{\lambda} D\right)$.

\subsubsection{Calcul des vecteurs propres}

Pour $\lambda$ valeur propre de l'opérateur $A_{p}$ les vecteurs propres associés sont donnés par $(12)$ où $\left(\alpha_{1}, \alpha_{2}\right)$ satisfait (11).

En résolvant numériquement le système $\frac{\mathrm{d} V}{\mathrm{~d} y}=M(y, p, \lambda) V$, avec la condition $V(L)=(0,0,1,0)(\mathrm{resp} . V(L)=$ $(0,0,0,1))$ on obtient $R_{11}(y, L, p, \lambda)=v_{1}(y), R_{21}(y, L, p, \lambda)=v_{4}(y), D_{11}(0, L, p, \lambda)=v_{3}(0), D_{21}(0, L, p, \lambda)=$ $v_{4}(0)\left(\operatorname{resp} . R_{12}(y, L, p, \lambda)=v_{1}(y), R_{22}(y, L, p, \lambda)=v_{2}(y), D_{12}(0, L, p, \lambda)=v_{3}(0), D_{22}(0, L, p, \lambda)=v_{4}(0)\right)$.

On calcule ensuite $\left(\alpha_{1}^{\prime}, \alpha_{2}^{\prime}\right)$ puis $\mathbf{u}$.

\section{Relation de Dispersion dans le CAS homogène ET DÉmonstration DES THÉORÈMES 1.1 ET 1.2}

Les fonctions $\underline{\rho}, \underline{\lambda}$ et $\underline{\mu}$ sont maintenant constantes. Nous allons d'abord calculer explicitement les fonctions $D_{i j}$ et $D$.

Posons

$$
c_{T}=\left(\frac{\underline{\mu}}{\underline{\rho}}\right)^{1 / 2}, \quad c_{L}=\left(\frac{\underline{\lambda}+2 \underline{\mu}}{\underline{\rho}}\right)^{1 / 2},
$$

qui sont, respectivement, la vitesse de propagation des ondes transversales (ondes $S$ ) et celle des ondes longitudinales (ondes $P$ ).

On vérifie aisément que si deux milieux élastiques caractérisés, respectivement par $\left\{\underline{\rho}_{1}, \underline{\lambda}_{1}, \underline{\mu}_{1}\right\}$ et $\left\{\underline{\rho}_{2}, \underline{\lambda}_{2}, \underline{\mu}_{2}\right\}$, sont tels que

$$
\frac{\underline{\mu}_{1}}{\underline{\lambda}_{1}+2 \underline{\mu}_{1}}=\frac{\underline{\mu}_{2}}{\underline{\lambda}_{2}+2 \underline{\mu}_{2}},
$$

alors les valeurs propres d'un opérateur réduit correspondant au deuxième milieu s'obtiennent en multipliant celles du premier par $\underline{\mu}_{2} / \underline{\mu}_{1}$, et il en est donc de même pour les courbes de dispersion. Les propriétés de ces dernières dépendent donc du rapport

$$
\kappa=\frac{\underline{\mu}}{\underline{\lambda}+2 \underline{\mu}}=\frac{c_{T}^{2}}{c_{L}^{2}}, \quad \text { où on a } 0<\kappa<1 / 2 .
$$

La matrice $M$ dans (6), donnée par (7), a maintenant des coefficients constants (i.e. indépendants de $y$ ) et on vérifie que ses valeurs propres sont $i k_{1},-i k_{1}, i k_{2}$ et $-i k_{2}$ où

$$
k_{1}=\left(\frac{\lambda}{c_{T}^{2}}-p^{2}\right)^{1 / 2}, \quad k_{2}=\left(\frac{\lambda}{c_{L}^{2}}-p^{2}\right)^{1 / 2} .
$$

La condition de Dirichet en $y=L$ fait que les opérateurs réduits n'ont pas zéro comme valeur propre et on peut alors supposer $\lambda \neq 0$. Ainsi les valeurs propres de $M$ sont distinctes deux à deux et la fonction $\mathbf{u}=\left(u_{1}, u_{2}\right)=\left(v_{1}, v_{2}\right)$ se met sous la forme

$$
\mathbf{u}=\left(\begin{array}{c}
d_{1} \\
d_{1}^{\prime}
\end{array}\right) \cos k_{1}(y-L)+\left(\begin{array}{c}
d_{2} \\
d_{2}^{\prime}
\end{array}\right) \frac{\sin k_{1}(y-L)}{k_{1}}+\left(\begin{array}{c}
d_{3} \\
d_{3}^{\prime}
\end{array}\right) \cos k_{2}(y-L)+\left(\begin{array}{c}
d_{4} \\
d_{4}^{\prime}
\end{array}\right) \frac{\sin k_{2}(y-L)}{k_{2}}
$$


où on convient que pour $k_{1}=0\left(\right.$ resp. $\left.k_{2}=0\right)$ on a $\left[\sin k_{1}(y-L)\right] / k_{1}=y-L\left(\operatorname{resp}\right.$. $\left.\left[\sin k_{2}(y-L)\right] / k_{2}=y-L\right)$.

Le fait que $\mathbf{u}$ vérifie le système différentiel (4), qui se réécrit

$$
\left\{\begin{array}{l}
\underline{\mu} \frac{\mathrm{d}^{2} u_{1}}{\mathrm{~d} y^{2}}+i p(\underline{\lambda}+\underline{\mu}) \frac{\mathrm{d} u_{2}}{\mathrm{~d} y}+\left(\underline{\rho} \lambda-(\underline{\lambda}+2 \underline{\mu}) p^{2}\right) u_{1}=0 \\
(\underline{\lambda}+2 \underline{\mu}) \frac{\mathrm{d}^{2} u_{2}}{\mathrm{~d} y^{2}}+i p(\underline{\lambda}+\underline{\mu}) \frac{\mathrm{d} u_{1}}{\mathrm{~d} y}+\left(\underline{\rho} \lambda-\underline{\mu} p^{2}\right) u_{2}=0
\end{array}\right.
$$

se traduit par :

$$
\begin{array}{cc}
d_{2}^{\prime}=-i p d_{1}, & d_{4}=i p d_{3}^{\prime}, \\
k_{1}^{2} d_{1}^{\prime}=i p d_{2}, & -i p d_{4}^{\prime}=k_{2}^{2} d_{3} .
\end{array}
$$

La condition au bord (5) se traduit aussi par

$$
d_{1}+d_{3}=0, \quad d_{1}^{\prime}+d_{3}^{\prime}=0 .
$$

Comme $\lambda$ est non nulle et que $p$ et $k_{1}$ ne s'annulent pas ensemble (même chose pour $p$ et $k_{2}$ ) on déduit, en utilisant (24), qu'il existe deux nombres $t_{1}$ et $t_{2}$ tels que

$$
\begin{array}{ll}
d_{2}=k_{1}^{2} t_{1}, & d_{1}^{\prime}=i p t_{1}, \\
d_{3}=-i p t_{2}, & d_{4}^{\prime}=k_{2}^{2} t_{2} .
\end{array}
$$

Finalement, en tenant compte de $(23,25)$, il vient

$$
\begin{array}{ll}
d_{3}^{\prime}=-d_{1}^{\prime}=-i p t_{1}, & d_{4}=i p d_{3}^{\prime}=p^{2} t_{1}, \\
d_{1}=-d_{3}=i p t_{2}, & d_{2}^{\prime}=-i p d_{1}=p^{2} t_{2} .
\end{array}
$$

Posons maintenant

$$
\begin{aligned}
& D_{11}^{\prime}(y, L, p, \lambda)=\left(k_{1}^{2}-p^{2}\right) \cos k_{1}(y-L)+2 p^{2} \cos k_{2}(y-L), \\
& D_{12}^{\prime}(y, L, p, \lambda)=i p\left(-\left(k_{1}^{2}-p^{2}\right) \frac{\sin k_{1}(y-L)}{k_{1}}+2 k_{2}^{2} \frac{\sin k_{2}(y-L)}{k_{2}}\right), \\
& D_{21}^{\prime}(y, L, p, \lambda)=-i p\left(2 k_{1}^{2} \frac{\sin k_{1}(y-L)}{k_{1}}-\left(k_{1}^{2}-p^{2}\right) \frac{\sin k_{2}(y-L)}{k_{2}}\right), \\
& D_{22}^{\prime}(y, L, p, \lambda)=2 p^{2} \cos k_{1}(y-L)+\left(k_{1}^{2}-p^{2}\right) \cos k_{2}(y-L) .
\end{aligned}
$$

En utilisant l'expression de la fonction $u$ ainsi que celles des coefficients $d_{i}, d_{i}^{\prime}, i=1, \ldots, 4$, données précédemment, on vérifie directement que

$$
\begin{aligned}
& v_{3}(y)=\underline{\mu}\left(i p u_{2}+\frac{\mathrm{d} u_{1}}{\mathrm{~d} y}\right)=\underline{\mu}\left(D_{11}^{\prime}(y, L, p, \lambda) t_{1}+D_{12}^{\prime}(y, L, p, \lambda) t_{2}\right), \\
& v_{4}(y)=(\underline{\lambda}+2 \underline{\mu}) \frac{\mathrm{d} u_{2}}{\mathrm{~d} y}+i \underline{\lambda} p u_{1}=\underline{\mu}\left(D_{21}^{\prime}(y, L, p, \lambda) t_{1}+D_{22}^{\prime}(y, L, p, \lambda) t_{2}\right) .
\end{aligned}
$$

Il est alors clair que la solution $V$ du système (6) qui prend la valeur $(0,0,1,0)$ en $y=L$ (celle-ci correspond en fait à $t_{2}=0$ ) est telle que

$$
v_{3}(y)=D_{11}(y, L, p, \lambda)=\frac{1}{\underline{\rho} \lambda} D_{11}^{\prime}(y, L, p, \lambda), \quad v_{4}(y)=D_{21}(y, L, p, \lambda)=\frac{1}{\underline{\rho} \lambda} D_{21}^{\prime}(y, L, p, \lambda) .
$$


Et celle qui prend $(0,0,0,1)$ en $y=L$ (elle correspond à $\left.t_{1}=0\right)$ est telle que

$$
v_{3}(y)=D_{12}(y, L, p, \lambda)=\frac{1}{\underline{\rho} \lambda} D_{12}^{\prime}(y, L, p, \lambda), \quad v_{4}(y)=D_{22}(y, L, p, \lambda)=\frac{1}{\underline{\rho} \lambda} D_{22}^{\prime}(y, L, p, \lambda) .
$$

On vérifie directement que

$$
\begin{aligned}
D(p, \lambda)=\operatorname{det}\left(D_{i j}(0, L, p, \lambda)\right)= & \frac{1}{(\underline{\rho} \lambda)^{2}}\left[\left(\left(k_{1}^{2}-p^{2}\right)^{2}+4 p^{4}\right) \cos \left(k_{1} L\right) \cos \left(k_{2} L\right)\right. \\
& \left.+4 p^{2}\left(k_{1}^{2}-p^{2}\right)-p^{2}\left(\left(k_{1}^{2}-p^{2}\right)^{2}+4 k_{1}^{2} k_{2}^{2}\right) \frac{\sin \left(k_{1} L\right)}{k_{1}} \frac{\sin \left(k_{2} L\right)}{k_{2}}\right] .
\end{aligned}
$$

Remarquons que nous retrouvons explicitement les propriétés de symétrie et d'antisymétrie des fonctions $D_{i j}$ par rapport à $p=0$ qui ont été démontrées dans le cas général.

Dans ce qui suit nous posons $D_{i j}^{\prime}(p, \lambda)=D_{i j}^{\prime}(0, L, p, \lambda)$ et $D^{\prime}(p, \lambda)=(\underline{\rho} \lambda)^{2} D(p, \lambda)$. Comme $\lambda$ est supposée non nulle on vérifie facilement que la fonction $D^{\prime}$ satisfait la propriété donnée dans le théorème 1.3, à savoir que pour tout $p$ la multiplicité d'une valeur propre de $A_{p}$ est égale à sa multiplicité comme racine de la fonction $D^{\prime}(p, \cdot)$.

En remplaçant les variables $\lambda$ et $p$ par $L^{2} \lambda / c_{T}^{2}$ et $L p$, respectivement, il est clair que l'étude de la relation de dispersion et des courbes de dispersion se ramène à celle du cas particulier, que nous considérons à partir de maintenant, dans lequel $L=1, c_{T}=1$ et donc $c_{L}=\kappa^{-1 / 2}$.

La fonction $D^{\prime}$ dépend aussi de $\kappa$ et on notera $D^{\prime}(p, \lambda, \kappa)$ pour préciser cette dépendance si nécessaire.

\subsection{Démonstration du théorème $\mathbf{1 . 1}$}

Les valeurs propres $\lambda$ de l'opérateur $A_{0}$ sont déterminées, à partir de la relation de dispersion, par :

$$
\cos (\sqrt{\lambda}) \cos (\sqrt{\kappa \lambda})=0
$$

et sont donc données explicitement par :

$$
\left\{\left(\frac{\pi}{2}+n \pi\right)^{2} ; \quad n \in \mathbb{Z}\right\} \cup\left\{\frac{1}{\kappa}\left(\frac{\pi}{2}+n^{\prime} \pi\right)^{2} ; \quad n^{\prime} \in \mathbb{Z}\right\}
$$

Les valeurs propres doubles de $A_{0}$ sont celles pour lesquelles $D_{i j}^{\prime}(0, \lambda)=0,1 \leq i, j \leq 2$, ce qui équivaut à

$$
\cos (\sqrt{\lambda})=0 \quad \text { et } \quad \cos (\kappa \sqrt{\lambda})=0 .
$$

On vérifie alors qu'elles sont données explicitement par les éléments

$$
\left\{\left(\frac{\pi}{2}+n \pi\right)^{2} ; n \in \mathbb{Z},\left(\frac{1}{2}+n\right) \kappa^{1 / 2}-\frac{1}{2} \in \mathbb{Z}\right\}
$$

À l'aide de logiciels mathématiques de calcul formel tels que Mathematica on peut calculer explicitement les dérivées partielles de la fonction $D^{\prime}$. Les expressions trouvées sont très compliquées et leur exploitation en vue d'une résolution explicite est impossible en général. Dans le cas particulier où $p=0$ et $\lambda$ une valeur propre double de $A_{0}$, ce que nous supposons maintenant, ces expressions se simplifient énormément. 
D'après les résultats généraux démontrés précédemment, on devrait avoir

$$
\partial_{p} D^{\prime}(0, \lambda)=\partial_{\lambda p}^{2} D^{\prime}(0, \lambda)=0, \quad(\text { par raison de symétrie) }
$$

et

$$
\partial_{\lambda} D^{\prime}(0, \lambda)=0, \text { avec } \partial_{\lambda}^{2} D^{\prime}(0, \lambda) \neq 0 \quad(\text { car } \lambda \text { est une valeur propre double })
$$

Les calculs explicites ci-dessous confirment ceci.

$$
\begin{aligned}
& \partial_{p} D^{\prime}(0, \lambda)=\partial_{\lambda} D^{\prime}(0, \lambda)=\partial_{\lambda p}^{2} D^{\prime}(0, \lambda)=0 \\
& \partial_{\lambda}^{2} D^{\prime}(0, \lambda)=\frac{\sqrt{\kappa} \lambda}{2} \sin (\sqrt{\lambda}) \sin (\sqrt{\kappa \lambda}) \\
& \partial_{p}^{2} D^{\prime}(0, \lambda)=2 \lambda\left(4-\frac{1+4 \kappa}{\sqrt{\kappa}} \sin (\sqrt{\lambda}) \sin (\sqrt{\kappa \lambda})\right) .
\end{aligned}
$$

Comme $\cos (\sqrt{\lambda})=\cos (\sqrt{\kappa \lambda})=0$, alors $\epsilon:=\sin (\sqrt{\lambda}) \sin (\sqrt{\kappa \lambda})$ est égal à \pm 1 , et on a

$$
\begin{aligned}
& \partial_{\lambda}^{2} D^{\prime}(0, \lambda)=\epsilon \frac{\sqrt{\kappa} \lambda}{2} \neq 0, \\
& \partial_{p}^{2} D^{\prime}(0, \lambda)=-\frac{2 \epsilon \lambda}{\sqrt{\kappa}}(2 \sqrt{\kappa}-\epsilon)^{2}, \quad\left(\operatorname{car} \epsilon^{2}=1\right) .
\end{aligned}
$$

Sachant que $\lambda$ est une valeur propre double de $A_{0}$ il existe deux courbes de dispersion $\lambda_{n_{1}}$ et $\lambda_{n_{2}}$ passant par $\lambda$ et, comme dans (22), on a

$$
\lambda_{n_{1}}^{\prime}(0)=\frac{2}{\sqrt{\kappa}}|2 \sqrt{\kappa}-\epsilon|, \quad \lambda_{n_{2}}^{\prime}(0)=-\frac{2}{\sqrt{\kappa}}|2 \sqrt{\kappa}-\epsilon| .
$$

Si $2 \sqrt{\kappa}-\epsilon \neq 0$ alors $\lambda_{n_{2}}^{\prime}(0)<0$ et on déduit que $\lambda_{n_{2}}$ n'est pas monotone sur $\mathbb{R}_{+}$. Ceci est donc toujours le cas si $\kappa \neq 1 / 4$ ou si $\sin (\sqrt{\lambda}) \sin (\sqrt{\lambda \kappa})=-1$.

Les valeurs exactes des dérivées dans le cas particulier (voir la Fig. 1) $\kappa=1 / 9$ et $\lambda_{n_{1}}(0)=\lambda_{n_{2}}(0)=(3 \pi / 2)^{2}$ (qui est une valeur propre double) sont

$$
\lambda_{n_{1}}^{\prime}(0)=10, \quad \lambda_{n_{2}}^{\prime}(0)=-10
$$

Ceci montre donc que les courbes de dispersion ne sont pas toujours croissantes sur $\mathbb{R}_{+}$.

La non monotonie sur $\mathbb{R}_{+}$des courbes de dispersion $\lambda_{n}$ n'est pas seulement liée au fait que $\lambda_{n}(0)$ soit une valeur propre double, comme on peut le penser. L'exemple numérique suivant illustre cette affirmation.

On prend $\rho(y)=2-y$ et $\underline{\lambda}(y)=\mu(y)=1+y$ pour tout $y \in(0,1)$, et on trace $(c f$. Fig. 2) une courbe de dispersion $\lambda_{n}$ avec $\lambda_{n}(0)$ une valeur propre simple de $A_{0}$. Nous utilisons pour cela la méthode numérique proposée dans la section précédente, et nous vérifions que $\lambda_{n}(0)$ est une valeur propre simple en calculant la quantité $\partial_{\lambda} D^{\prime}\left(0, \lambda_{n}(0)\right)$ et en constatant qu'elle n'est pas nulle.

\subsection{Démonstration du théorème $\mathbf{1 . 2}$}

Le paramètre $\kappa$ est maintenant variable, aussi nous noterons $A_{0}^{\kappa}$ l'opérateur réduit en $p=0$ et $\lambda_{n}^{\kappa}, n \geq 1$, les courbes de dispersion associées. Les valeurs propres de $A_{0}^{\kappa}$ sont les solutions $\theta$ de $\cos (\sqrt{\theta}) \cos (\sqrt{\kappa \theta})=0$. Ainsi les éléments $\theta$ de

$$
\left\{\left(\frac{\pi}{2}+2 n \pi\right)^{2} ; \quad n \in \mathbb{Z}\right\}
$$




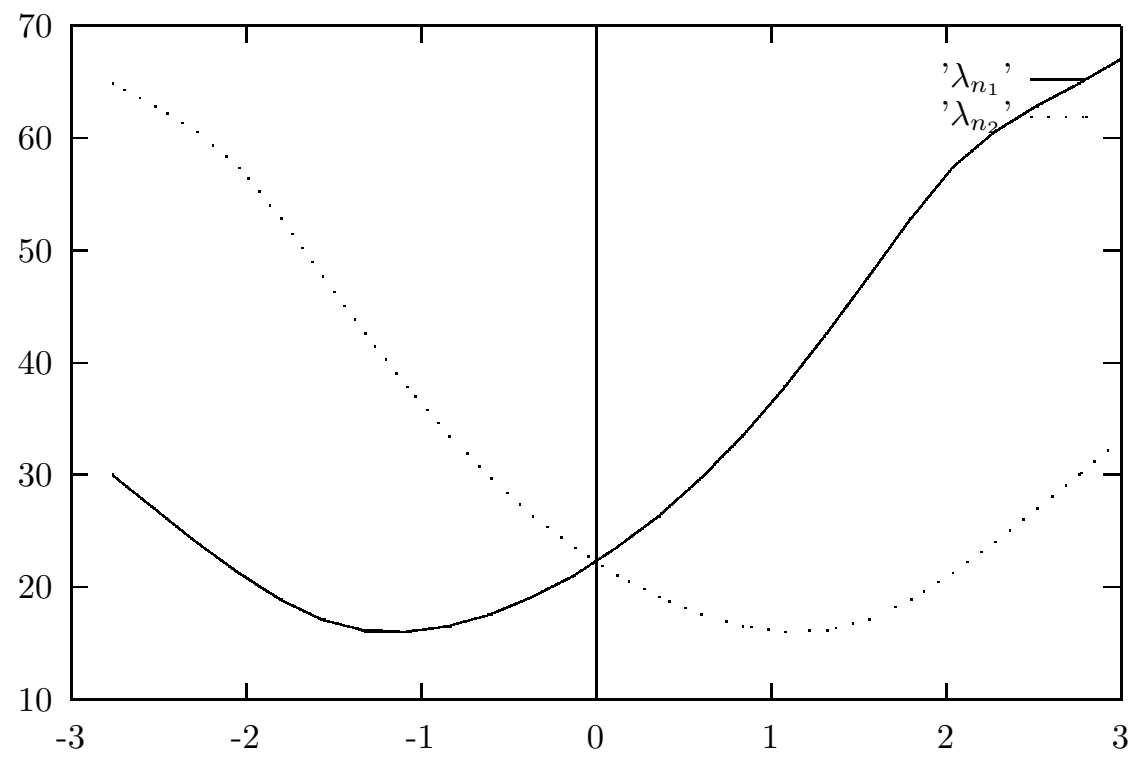

Figure 1. Les fonction $\lambda_{n_{1}}$ et $\lambda_{n_{2}}$ sont symétriques l'une de l'autre et ont une dérivée non nulle en 0 .

sont des valeurs propres de $A_{0}^{\kappa}$, pour tout $\kappa$, satisfaisant $\cos (\sqrt{\theta})=0$ et $\sin (\sqrt{\theta})=1$.

On sait que $\left\{\mathrm{e}^{i \sqrt{\kappa} 2 n \pi} ; n \in \mathbb{Z}\right\}$ est dense dans la sphère unité $\left\{\mathrm{e}^{i \alpha} ; \alpha \in[0,2 \pi]\right\}$ si $\sqrt{\kappa}$ est un nombre irrationnel. Fixons un nombre irrationnel $\kappa_{1}$ dans $(0,1 / 4)$. On peut donc extraire une sous-suite $\theta_{j}, j \geq 1$, de $(26)$, satisfaisant

$$
\cos \left(\sqrt{\theta_{j}}\right)=0, \quad \sin \left(\sqrt{\theta_{j}}\right)=1,
$$

et

$$
\cos \left(\sqrt{\kappa_{1} \theta_{j}}\right) \rightarrow 1, \text { lorsque } j \rightarrow+\infty
$$

Montrons qu'il existe $\kappa_{0} \in\left(0, \kappa_{1}\right)$ et $j_{0}$ assez grand tels qu'on ait

$$
\cos \left(\sqrt{\kappa_{0} \theta_{j_{0}}}\right) \neq 0, \quad \partial_{p}^{2} D^{\prime}\left(0, \theta_{j_{0}}, \kappa_{0}\right)=0
$$

(la fonction $D^{\prime}$ étant paire par rapport à la variable $p$ on a $\left.\partial_{p} D^{\prime}\left(0, \theta_{j_{0}}, \kappa_{0}\right)=0, \partial_{p}^{3} D^{\prime}\left(0, \theta_{j_{0}}, \kappa_{0}\right)=0\right)$. Notons $\lambda_{n_{j}}^{\kappa_{0}}, j \geq 1$, les courbes de dispersion satisfaisant $\lambda_{n_{j}}^{\kappa_{0}}(0)=\theta_{j}$. Le fait que $\cos \left(\sqrt{\kappa_{0} \theta_{j_{0}}}\right) \neq 0$ implique que $\lambda_{n_{j_{0}}}^{\kappa_{0}}(0)$ est une valeur propre simple de $A_{0}^{\kappa_{0}}$. En appliquant les résultats de caractérisation des seuils à l'aide de la

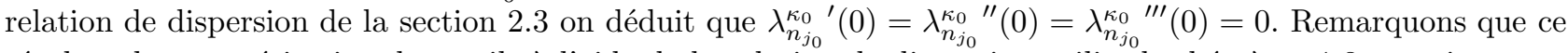
résultat de caractérisation des seuils à l'aide de la relation de dispersion utilise le théorème 1.3 ce qui montre son importance.

Nous vérifions à l'aide de Mathematica que pour tout $\kappa \in(0,1 / 2)$ on a

$$
\begin{aligned}
d_{j}(\kappa) & :=\partial_{p}^{2} D^{\prime}\left(0, \theta_{j}, \kappa\right) \\
& =\theta_{j}\left(8+\sqrt{\theta_{j}} \cos \left(\sqrt{\kappa \theta_{j}}\right)-2 \frac{1+4 \kappa}{\sqrt{\kappa}} \sin \left(\sqrt{\kappa \theta_{j}}\right)\right) .
\end{aligned}
$$




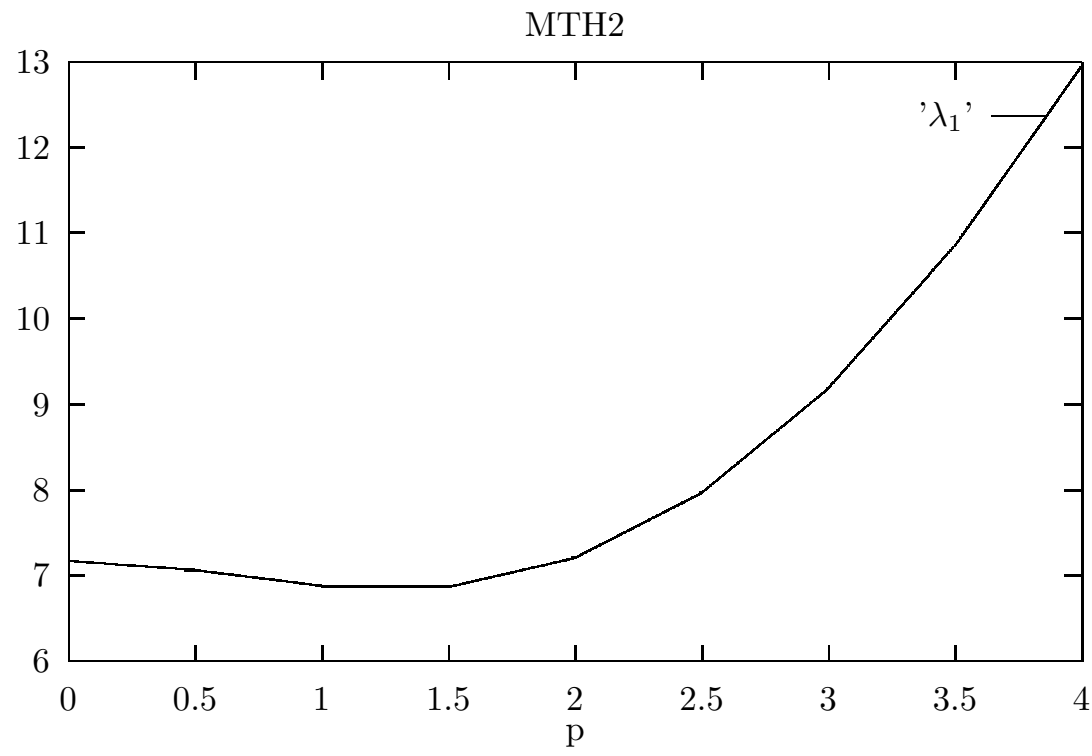

FiguRE 2. La fonction $\lambda_{n}$ est non monotone sur $\mathbb{R}_{+}$alors que $\lambda_{n}(0)$ est une valeur propre simple.

La fonction $d_{j}$ ainsi définie est de classe $C^{\infty}(\mathbb{R})$ et on a

$$
d_{j}(0)=\theta_{j}\left(8-\sqrt{\theta_{j}}\right) .
$$

Comme $\theta_{j} \rightarrow+\infty$, lorsque $j \rightarrow+\infty$, et en tenant compte de (27), on voit que

$$
d_{j}(0) \rightarrow-\infty, \quad d_{j}\left(\kappa_{1}\right) \rightarrow+\infty .
$$

Pour $j$ assez grand, on a donc $d_{j}(0)<0$ et $d_{j}\left(\kappa_{1}\right)>0$. Le théorème des valeurs intermédiaires donne l'existence de $\kappa_{0} \in\left(0, \kappa_{1}\right)$ tel que $d_{j}\left(\kappa_{0}\right)=\partial_{p}^{2} D^{\prime}\left(0, \theta_{j}, \kappa_{0}\right)=0$. On a aussi $\cos \left(\sqrt{\kappa_{0} \lambda_{n_{j}}(0)}\right) \neq 0$. En effet, supposant le contraire, on aurait

$$
8-2 \frac{1+4 \kappa_{0}}{\sqrt{\kappa_{0}}} \sin \left(\sqrt{\kappa_{0} \theta_{j}}\right)=0
$$

$\operatorname{avec}\left|\sin \left(\sqrt{\kappa_{0} \theta_{j}}\right)\right|=1$, d'où $\kappa_{0}=1 / 4$, ce qui contredirait $\kappa_{0}<\kappa_{1}<1 / 4$.

Note des auteurs. Depuis la soumission de ce travail nous nous sommes rendus compte de l'existence de représentations graphiques montrant des courbes de dispersion non monotones (avec la condition de surface libre sur les deux bords de la bande) mais sans que cette particularité soit relevée ni, a fortiori, étudiée ( $c f$. B.A. Auld, Acoustic Fields and Waves in Solids, Vol. II, Krieger (1990), pp. 80, 82).

\section{REFERENCES}

[1] J.D. Achenbach, Wave propagation in elastic solids. North Holland (1973).

[2] A. Bamberger, Y. Dermenjian et P. Joly, Mathematical analysis of the propagation of elastic guided waves in heterogeneous media. J. Differential Equations 88 (1990) 113-154.

[3] T. Bouhennache, Analyse spectrale d'une bande élastique isotrope stratifiée et applications. C.R. Acad. Sci. Sér. I 326 (1998) 641-644. 
[4] T. Bouhennache, Spectral analysis of an isotropic stratified elastic strip and applications. Osaka J. Math. (à paraître).

[5] T. Bouhennache, Étude d'une bande élastique multistratifiée: étude spectrale et principe d'absorption limite. Thèse de doctorat, Université de Provence (1997).

[6] E.A. Coddington et N. Levinson, Theory of ordinary differential equations. Int. Series in Pure and Appl. Math., McGraw-Hill Book Company (1955).

[7] E. Croc et Y. Dermenjian, Analyse spectrale d'une bande acoustique multistratifiée : Principe d'absorption limite pour une stratification simple. SIAM J. Math. Anal. 26 (1995) 880-924.

[8] L. Hörmander, The Analysis of Linear Partial Differential Operators, II. Springer-Verlag (1983).

[9] T. Kato, Perturbation theory for linear operators. Classics in Mathematics, Springer-Verlag (1995).

[10] S. Lang, Analyse réelle. InterEditions (1977).

[11] B. Malgrange, Existence et approximation des solutions des équations aux dérivées partielles et des équations de convolution. Ann. Inst. Fourier (Grenoble) 6 (1955-56) 271-355.

[12] R. Skalak. J. Appl. Mech. 24 (1957) 59. 